\title{
Neutron scattering study of the magnetic microstructure of nanocrystalline gadolinium
}

\author{
Frank Döbrich * \\ Experimentalphysik, Universität des Saarlandes, Postfach 151150, D-66041 Saarbrücken, Germany and Laboratory for the Physics of \\ Advanced Materials, University of Luxembourg, 162A Avenue de la Faïencerie, L-1511 Luxembourg, Luxembourg \\ Joachim Kohlbrecher \\ Paul Scherrer Institut, CH-5232 Villigen PSI, Switzerland \\ Melissa Sharp ${ }^{\dagger}$ and Helmut Eckerlebe \\ HZG Research Center, Max-Planck-Straße 1, D-21502 Geesthacht, Germany \\ Rainer Birringer \\ Experimentalphysik, Universität des Saarlandes, Postfach 151150, D-66041 Saarbrücken, Germany \\ Andreas Michels \\ Experimentalphysik, Universität des Saarlandes, Postfach 151150, D-66041 Saarbrücken, Germany and Laboratory for the Physics of \\ Advanced Materials, University of Luxembourg, 162A Avenue de la Faïencerie, L-1511 Luxembourg, Luxembourg
}

(Received 5 December 2011; published 7 March 2012)

\begin{abstract}
We report grain-size-dependent results on nanocrystalline bulk Gd obtained by magnetic small-angle neutron scattering (SANS) and magnetometry. This approach allows one to study systematically how the magnetic microstructure of this rare-earth metal is affected by defects in the atomic microstructure, which are largely present in nanocrystalline materials, predominantly in the form of grain boundaries. The neutron scattering data reveal two types of angular anisotropies in the magnetic-field-dependent scattering cross section that are typically not seen in the coarse-grained polycrystal. In particular, a cloverleaf-shaped anisotropy and an elongation of the scattering pattern in the direction of the applied magnetic field have been detected. While the first result, which is an exceptional finding even in the nanocrystalline state, can be attributed to pronounced spin disorder in the vicinity of the Gd grain boundaries, the second anisotropy is related to spin misalignment due to the random magnetocrystalline anisotropy within the individual crystallites. Furthermore, we have calculated the correlation function of the spin misalignment from the radially averaged data, which gives access to the characteristic length scales on which the magnetization is perturbed by crystal defects. The results of this real-space analysis independently support the findings from magnetometry and field-dependent SANS. Wide-angle x-ray diffraction data indicate that stacking faults may limit the range of spin-misalignment fluctuations due to random anisotropy in this material.
\end{abstract}

DOI: 10.1103/PhysRevB.85.094411

PACS number(s): 75.25.-j, 61.05.fg, 75.50.Tt

\section{INTRODUCTION}

Nanostructured ferromagnetic solids receive strong interest in fundamental and applied science due to the fact that reducing the size of the microscopic building blocks of such systems strongly influences their macroscopic magnetic behavior. The impact of structural details, such as the shape of individual particles or the character of interfaces on the overall materials properties, is found to be equally high. ${ }^{1-3}$ In bulk magnetic nanomaterials, microstructural defects are largely present in the form of internal interfaces, such as phase or grain boundaries. The core region of these defects is characterized by lattice distortions and reduced crystal symmetry, which may lead to a local variation of the materials parameters. In this study, we focus on the role of such lattice imperfections for the magnetic behavior of the rare-earth element Gd.

The magnetism of Gd originates from the strongly localized electronic spins of the $4 f$ shell, which are indirectly coupled via the Ruderman-Kittel-Kasuya-Yosida interaction. ${ }^{4}$ Due to the absence of a $4 f$ angular momentum, Gd exhibits a comparatively low magnetocrystalline anisotropy and thus takes a prominent position among the rare-earth elements. ${ }^{5}$
As opposed to most other heavy rare-earth elements, Gd does not possess a helical antiferromagnetic phase and orders ferromagnetically below $293 \mathrm{~K}^{6}{ }^{6}$ Despite a long tradition of research on $\mathrm{Gd}$, there is still a strong interest today not only in the nature of the phase transition ${ }^{7-10}$ but also in questions related, e.g., to the electronic band structure of $\mathrm{Gd}^{11,12}$ the magnetothermal properties, ${ }^{13,14}$ and the origin of the magnetocrystalline anisotropy. ${ }^{15}$ Furthermore, the importance of microstructure, in particular, the impact of interfaces and/or confinement on the magnetic properties of $\mathrm{Gd}$, has been explored with increasing intensity. ${ }^{16-23}$

In order to relate the overall magnetic properties of a sample to its microstructure, it is vital to understand how lattice defects such as grain boundaries affect magnetism on a microscopic scale. Therefore, we have performed magnetic small-angle neutron scattering (SANS) measurements on polycrystalline Gd with an average crystallite size of about $20 \mathrm{~nm}$ in the as-prepared state. Such specimens are characterized by a high volume fraction of grain boundaries and a random orientation of the crystallographic axes of the individual crystallites. ${ }^{18}$ Due to the extraordinarily high absorption cross section of natural 
Gd for thermal and cold neutrons, only very few neutron scattering studies on Gd exist, ${ }^{24-28}$ mostly on single crystalline ${ }^{160} \mathrm{Gd}$. This low-capturing isotope has also been employed for the current SANS measurements. Despite the necessity to use this rather expensive material, the SANS method is virtually without alternative for obtaining the required information since it is able to probe the magnetic structure in the bulk of a sample (as opposed to surface-sensitive methods) and on the highly relevant micromagnetic length scale between about 1 and $100 \mathrm{~nm}$. The first results on a nanocrystalline ${ }^{160} \mathrm{Gd}$ sample with an average grain size of $21 \mathrm{~nm}$ have been reported in Refs. 29 and 30. As it is of great interest to investigate how changes in the atomic microstructure are reflected (i) in the magnetic microstructure and (ii) in the macroscopic magnetic properties, we provide here grain-size-dependent SANS data on nanocrystalline Gd. The SANS measurements are complemented by the results of classical magnetometry.

This paper is organized as follows: In Sec. II we briefly summarize the experimental details of the present study. Section III provides an introduction to basic theoretical aspects of magnetic SANS as referred to in the following sections. In particular, these relate to the differential scattering cross section of a ferromagnetic solid and the correlation function of the spin misalignment. In Sec. IV we present the experimental results as obtained by the different methods, followed by a discussion of the data. This includes a microstructural characterization by $\mathrm{x}$-ray diffraction, temperature-dependent magnetometry, and substantial grain-size-dependent SANS results. Section V summarizes the main findings of this study.

\section{EXPERIMENT}

The nanocrystalline Gd samples have been prepared by the inert-gas condensation (igc) method, as described, e.g., in Ref. 31. In the igc process, Gd metal is evaporated from a tungsten boat under a He atmosphere (99.999\% purity) at a pressure of 2 mbar. The resulting nanoparticles are collected on a cold finger and compacted in situ at $1.4 \mathrm{GPa}$, yielding disk-shaped samples with a diameter of $8 \mathrm{~mm}$, a thickness of $0.2-1.0 \mathrm{~mm}$, and a mass density of $98 \%-99 \%$ (determined by the Archimedes method) as compared to the single-crystal value. ${ }^{6}$ Average grain size $D$ and inhomogeneous microstrain $\epsilon$ were estimated from wide-angle x-ray diffraction data by means of Williamson-Hall analysis. ${ }^{32,33}$ Typical values of these parameters in the as-prepared state are $D=15-25 \mathrm{~nm}$ and $\epsilon=0.3 \%-0.6 \%$. Coarsening of the grain structure was induced by annealing the specimens in a He-filled quartz tube. Further details on sample preparation and characterization can be found in Ref. 18. The magnetization measurements were carried out on a Quantum Design Physical Properties Measurement System (PPMS) extraction magnetometer.

The samples for the SANS experiments were prepared by employing the low-capturing isotope ${ }^{160} \mathrm{Gd}$ as starting material. The residual absorption cross section of $\sigma_{a}=641$ barns, as estimated from the atomic concentration of the individual isotopes $\left(0.2\right.$ at. $\%{ }^{155} \mathrm{Gd},{ }^{156} \mathrm{Gd}$, and ${ }^{157} \mathrm{Gd}$; 0.8 at. $\%{ }^{158} \mathrm{Gd}$; and 98.6 at. $\left.\%{ }^{160} \mathrm{Gd}\right),{ }^{34}$ ensured a sufficiently high sample transmission $\tau$, which is a prerequisite for the application of the SANS technique. ${ }^{35}$ Over most of the applied magnetic field range, the experimentally determined transmission shows very good agreement with the calculated values of $\tau=18 \%$ and $\tau=14 \%$, assuming a purely absorption-based transmission. As may be expected, the measured $\tau$ decreases at low magnetic fields, in accordance with an increased (magnetic) scattering contribution to the total attenuation of the primary neutron beam. ${ }^{36}$

The SANS experiments were performed at HZG Research Center Geesthacht, Germany, and at Paul Scherrer Institut, Switzerland, using an incident mean neutron wavelength of $\lambda=6.0 \AA$ with a wavelength broadening of $\Delta \lambda / \lambda=10 \%$ (FWHM). The data were corrected in the usual way for background scattering, detector efficiency, and solid-angle distortion and normalized to absolute units by means of a vanadium standard sample. ${ }^{37,38}$

\section{THEORETICAL BACKGROUND}

\section{A. SANS cross section}

As a versatile and powerful bulk method for the investigation of structural inhomogeneities on the scale of a few to a few hundred nanometers, the small-angle scattering technique is used for a wide range of problems, and we refer the reader to Refs. 39 and 40 for a general introduction. In this section, we focus on special aspects of magnetic SANS, as discussed in Ref. 41.

Using a Cartesian coordinate system with $\mathbf{e}_{x}$ along the axis of the incident neutron beam and $\mathbf{e}_{z}$ along the external magnetic field $\mathbf{H}$, the elastic differential SANS cross section $d \Sigma / d \Omega$ of a ferromagnet at scattering vector $\mathbf{q}$ reads $^{41}$

$$
\begin{aligned}
\frac{d \Sigma}{d \Omega}(\mathbf{q})= & \frac{8 \pi^{3}}{V}\left[|\widetilde{N}|^{2}+b_{H}^{2}\left|\widetilde{M}_{x}\right|^{2}+b_{H}^{2}\left|\tilde{M}_{y}\right|^{2} \cos ^{2} \theta\right. \\
& \left.+b_{H}^{2}\left|\tilde{M}_{z}\right|^{2} \sin ^{2} \theta-b_{H}^{2}\left(\widetilde{M}_{y} \tilde{M}_{z}^{*}+\tilde{M}_{y}^{*} \tilde{M}_{z}\right) \sin \theta \cos \theta\right] .
\end{aligned}
$$

In Eq. (1), $V$ is the scattering volume, $b_{\underset{H}{N}}=\underset{\sim}{2} .7 \times 10^{-15} \mathrm{~m} / \mu_{\widetilde{\sim}}$ ( $\mu_{B}$ is the Bohr magneton), and $\widetilde{N}, \widetilde{M}_{x}, \widetilde{M}_{y}$, and $\widetilde{M}_{z}$ denote, respectively, the Fourier transforms of the nuclear scattering-length density and of the Cartesian components of the magnetization. The asterisk $\left(^{*}\right)$ refers to the complex conjugated quantity, and $\theta$ is the angle between $\mathbf{q}$ and $\mathbf{H}$. Note that in addition to the intrinsically anisotropic nature of magnetic neutron scattering, which enters the SANS cross section via the trigonometric functions in Eq. (1), the Fourier coefficients of the nuclear and magnetic scattering-length densities depend explicitly on the scattering vector $\mathbf{q}$, which may also give rise to an anisotropic SANS signal.

\section{B. Correlation function of the spin misalignment}

The autocorrelation function of the spin misalignment can be defined as ${ }^{42}$

$$
C(\mathbf{r})=\frac{1}{M_{S}^{2} V} \iiint \mathbf{M}_{P}(\mathbf{x}) \mathbf{M}_{P}(\mathbf{x}+\mathbf{r}) d^{3} \mathbf{x},
$$

where $\mathbf{M}_{P}(\mathbf{x})=\mathbf{M}(\mathbf{x})-\langle\mathbf{M}\rangle$ is the difference between the local magnetization vector $\mathbf{M}(\mathbf{x})$ and the macroscopic mean magnetization $\langle\mathbf{M}\rangle . C(\mathbf{r})$ is related to the radially averaged 
spin-misalignment scattering cross section $d \Sigma_{M} / d \Omega$ by ${ }^{42}$

$$
C(r)=\frac{a}{2 \pi^{2} b_{m}^{2} \rho_{a}^{2} r} \int_{0}^{\infty} q \frac{d \Sigma_{M}}{d \Omega}(q) \sin (q r) d q .
$$

Here, $a$ is a numerical factor involved in the orientation average, $\rho_{a}$ denotes the atomic density, and $b_{m}=b_{H} \mu_{a}$ (with $\mu_{a}$ being the atomic magnetic moment) is the atomic magnetic scattering length. As an approximation to $d \Sigma_{M} / d \Omega$ at a particular magnetic field, we have subtracted $d \Sigma / d \Omega$ at the highest field of $\mu_{0} H_{\max }=5 \mathrm{~T}$ from the cross section at $H<H_{\max }$. This procedure also allows one to estimate the scattering contributions due to terms in Eq. (1) that contain $\widetilde{M}_{x}$ and $\widetilde{M}_{y} \cdot{ }^{43}$ A measure for the characteristic length scale over which perturbations in the spin structure decay is given by the correlation length $l_{C}$, which can be defined as the value of $r$ at which $C(r)=C(0) / e$. Here, $C(0)$ denotes the value of the correlation function at $r=0 .{ }^{44}$ Alternative approaches for the extraction of a correlation length $l_{C}$ from $C(r)$ data can be found, e.g., in Refs. 30 and 45.

The accessible range of scattering vectors in the SANS experiments was limited to $0.02 \mathrm{~nm}^{-1} \lesssim q \lesssim 1.5 \mathrm{~nm}^{-1}$. It is a well-known result of the theory of Fourier transformation that the maximum value of the momentum transfer $q_{\max }$ limits the resolution in real space to $\Delta r \gtrsim \pi / q_{\max } \cong 2 \mathrm{~nm}$ and that the size of the largest structure in the system is roughly given by $r_{\max } \lesssim \pi / q_{\min } \cong 150 \mathrm{~nm} .{ }^{39}$ The correlation function $C(r)$, Eq. (3), was obtained by means of direct Fourier transformation within $r_{\min }=2 \pi / q_{\max } \cong 4 \mathrm{~nm}$ and $r_{\max }$. For the numerical computation of $C(r)$ (in order to reduce termination effects), the experimental scattering data beyond $q_{\max }$ were extrapolated to infinity using Porod's law, $d \Sigma_{M} / d \Omega \propto q^{-4}$, and the extrapolation from $q_{\min }$ to $q=0$ was carried out using different schemes (linear and constant). Likewise, in order to estimate the uncertainties in the values of the correlation lengths, we have tested different procedures (limiting cases) for subtracting the SANS data at the highest experimental magnetic field of $5 \mathrm{~T}$ from the measurements at lower fields.

In principle, one may use Glatter's indirect Fouriertransformation method ${ }^{39}$ for the computation of $C(r)$. However, this technique, which has been originally developed for particle scattering, requires the rather precise knowledge of the maximum particle size. ${ }^{39}$ Since the magnetic SANS investigated in this study has its origin in continuously varying magnetic-field-dependent magnetization profiles (no sharp boundaries), we have employed the direct Fourier transformation technique for obtaining rough information on the characteristic real-space length scales.

\section{RESULTS AND DISCUSSION}

While the measurement of the macroscopic magnetization reduces the information contained in the magnetic microstructure of a sample to a single volume-averaged scalar value, the SANS method gives access to a vastly richer set of data, i.e., to the Fourier spectrum of correlated spatial magnetization fluctuations. However, due to the experimental limitations of a SANS instrument, the real-space information that can be obtained is restricted to a range between a few and a few hundred nanometers. Consequently, classical magnetization

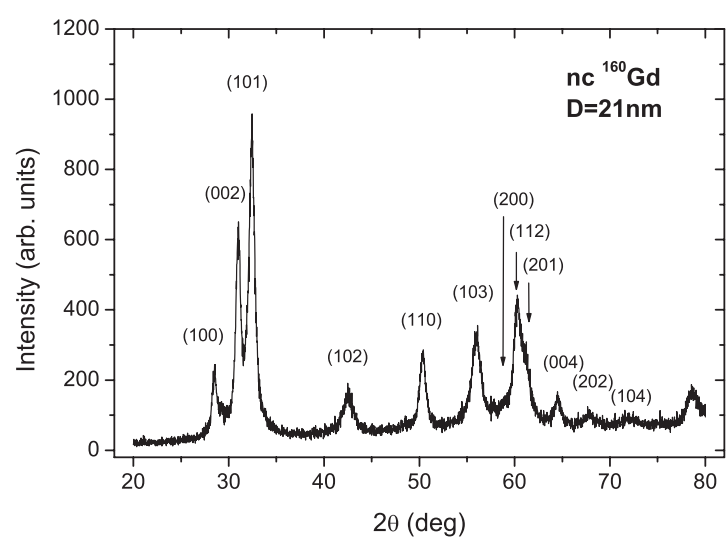

FIG. 1. X-ray diffraction scan of nanocrystalline ${ }^{160} \mathrm{Gd}$ (average grain size $D=21 \mathrm{~nm}$ ).

measurements that complement the SANS data have been performed (see Sec. IV B). The results of the microstructural characterization by means of x-ray diffraction are summarized in the following section.

\section{A. X-ray diffraction}

As a standard method for the characterization of finegrained materials with respect to crystallite size, inhomogeneous microstrain, or stacking-fault densities, $\mathrm{x}$-ray diffraction (XRD) is widely used since it allows both fast and nondestructive measurements. ${ }^{33,46}$ Figure 1 shows the XRD scan of an as-prepared nanocrystalline ${ }^{160} \mathrm{Gd}$ sample. All peak positions are found to be consistent with hcp Gd. Furthermore, the relative intensities agree well with the fact that these samples are isotropic polycrystals, as was previously found from texture measurements. ${ }^{47}$ Details of the sample characterization regarding grain size and microstrain can be found in Refs. 18 and 32. In the case of nanocrystalline Gd, stacking-fault densities are difficult to access from a conventional XRD scan, as data evaluation of the Warren-Averbach type is hindered by a considerable background and tight peak positions. Therefore, in order to be able to estimate values for the stackingfault densities $\alpha$ and $\beta$, corresponding to deformation faults and growth faults, respectively, we have adapted a method described by Warren ${ }^{46}$ based on the FWHM breadth of selected peaks (see Appendix B). The results of the XRD analysis for the two ${ }^{160} \mathrm{Gd}$ samples employed in the SANS measurements are summarized in Table I. A significant stacking-fault density with respect to the experimental uncertainty was only detected in sample B. Furthermore, large values for the inhomogeneous microstrain $\epsilon$ are found in both samples.

\section{B. Magnetometry}

Grain-size-dependent magnetization isotherms of $\mathrm{Gd}$ at $T=5 \mathrm{~K}$ are displayed in Fig. 2(a). It is seen that a reduction of the grain size $D$ results in a considerable decrease of the macroscopic mean magnetization with respect to the coarse-grained state; e.g., at $\mu_{0} H=1 \mathrm{~T}$, we find a relative reduction of about $20 \%$ for the smallest grain size of $14 \mathrm{~nm}$. Even at fields as high as $9 \mathrm{~T}$, the effect of nanocrystallinity on the macroscopic magnetization is still significant. By stepwise annealing the nanocrystalline samples to the coarse-grained 
TABLE I. Results of the XRD analysis of the nanocrystalline ${ }^{160} \mathrm{Gd}$ samples A and B used for the neutron experiments. Coarsening of the grain microstructure of sample B was induced by annealing at temperatures $T_{a}=150^{\circ} \mathrm{C}$ (sample denoted as B') and $200^{\circ} \mathrm{C}$ (B"). The quantities $\alpha$ and $\beta$ denote deformation and growth faults, respectively. ${ }^{46}$ The average distance between two faults (in nm) has been estimated as $L_{\alpha}=d_{002} / \alpha$ and $L_{\beta}=d_{002} / \beta$, respectively, where $d_{002}$ is the distance of adjacent atomic layers in the $c$ direction. ${ }^{6,48}$ In the last two columns, the results for the average grain size $D$ and the microstrain $\epsilon$ are listed.

\begin{tabular}{lccrrcc}
\hline \hline & $\alpha(\%)$ & $\beta(\%)$ & $L_{\alpha}$ & $L_{\beta}$ & $D(\mathrm{~nm})$ & $\epsilon(\%)$ \\
\hline A & $2.1 \pm 2.7$ & $1.5 \pm 3.8$ & 14 & 19 & 21 & $0.45 \pm 0.22$ \\
B & $0.9 \pm 0.9$ & $3.7 \pm 1.4$ & 32 & 8 & 25 & $0.35 \pm 0.08$ \\
B & $1.2 \pm 1.4$ & $1.9 \pm 1.9$ & 24 & 15 & 35 & $0.09 \pm 0.01$ \\
B” & $-0.2 \pm 0.3$ & $1.2 \pm 0.5$ & & 24 & 95 & $0.06 \pm 0.01$ \\
\hline \hline
\end{tabular}

state, we "recover" the single-crystal saturation magnetization value of $\mu_{0} M_{S}=2.69 \mathrm{~T}$, and a scaling law for the relative magnetization reduction $\Delta M / M \propto D^{-1}$ is found (see Fig. 3). This is particularly remarkable since the volume fraction of grain boundaries follows approximately the same grain-size dependence. $^{49}$ In Appendix A we give an estimation for $\Delta M / M$ based on the assumption of a reduced magnetization within the grain-boundary phase, which yields the above $D^{-1}$
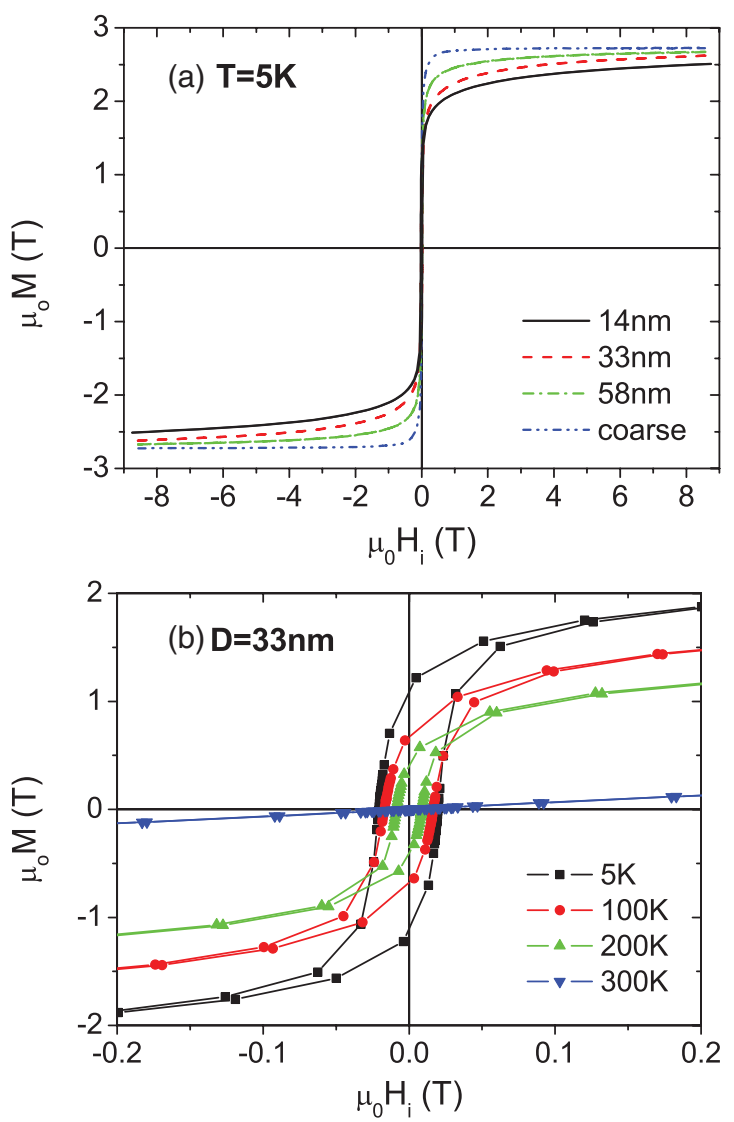

FIG. 2. (Color online) (a) Grain-size-dependent magnetization isotherms of $\mathrm{Gd}$ at $T=5 \mathrm{~K}$. The coarse-grained reference sample was obtained by annealing from the nanocrystalline as-prepared state. (b) Hysteresis loops of the $D=33 \mathrm{~nm}$ sample for temperatures of $T=5,100,200$, and $300 \mathrm{~K}$. Lines are guides to the eyes.

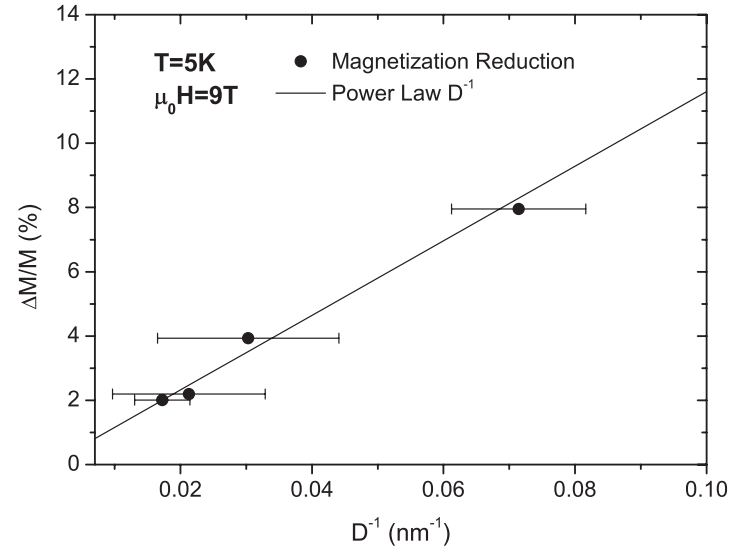

FIG. 3. Relative reduction $\Delta M / M:=\left(M_{\mathrm{cg}}-M_{\mathrm{nc}}\right) / M_{\mathrm{cg}}$ (dots) of the macroscopic magnetization as a function of the average grain size $D$ at $\mu_{0} H=9 \mathrm{~T}$ and $T=5 \mathrm{~K}$ (data taken from Ref. 30). Note that $D$ is displayed on a reciprocal scale.

law. Note that density fluctuations are (i) too small to provide a quantitative explanation and (ii) do not contribute to $\Delta M / M$ as shown in Fig. 3 since the mass magnetization was used (see Appendix A). Therefore, we suggest that incomplete saturation due to spin disorder within the grain-boundary region may be responsible for the observed $D^{-1}$ behavior. In other words, these results indicate the presence of a reduced effective magnetization in the grain-boundary phase, which may, e.g., be a consequence of competing or frustrated interactions between the $4 f$ moments. The estimated value for the relative reduction of the grain-boundary magnetization (at $9 \mathrm{~T}$ ) with respect to the bulk of the grains amounts to $26 \%$.

The effect of temperature on the hysteresis loop is displayed in Fig. 2(b) for a grain size of $D=33 \mathrm{~nm}$. The data exhibit the usual reduction of remanence and coercivity with increasing temperature. As may be expected from the single-crystal value $T_{C}=293 \mathrm{~K}$ for the Curie temperature of $\mathrm{Gd},{ }^{6}$ the data measured at the highest temperature $T=300 \mathrm{~K}$ do not show a hysteresis. Note also that $T_{C}$ is additionally shifted toward lower $T$ in the nanocrystalline material. ${ }^{18}$

A detailed measurement of the coercive field $H_{C}$ as a function of temperature (see Fig. 4) shows a local minimum between 10 and $20 \mathrm{~K}$ for grain sizes of 14 and $33 \mathrm{~nm}$, which

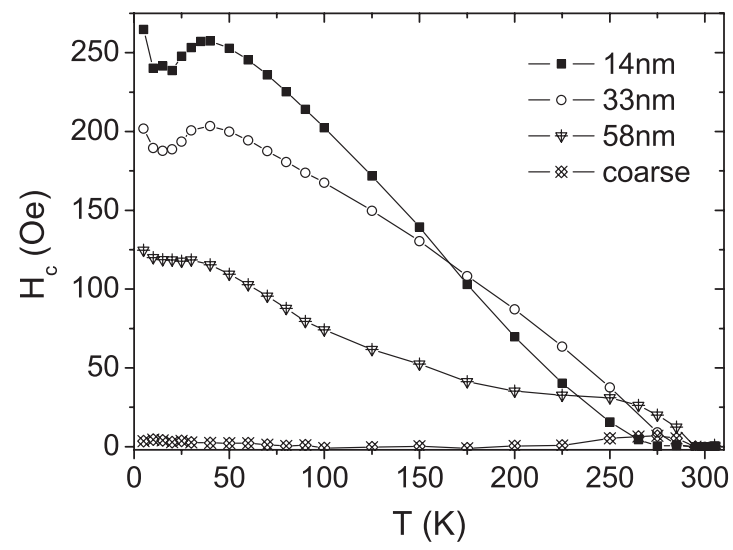

FIG. 4. Grain-size and temperature dependence of the coercive field $H_{C}$ of $\mathrm{Gd}$. Lines are guides to the eyes. 
is seen to vanish with coarsening of the grain microstructure. Regarding the grain-size dependence of $H_{C}$, it is seen that, below about $175 \mathrm{~K}$, the coercivity is strongly reduced with increasing grain size. At higher temperatures (e.g., at $250 \mathrm{~K}$ ), $H_{C}$ initially increases with increasing grain size, goes through a maximum, and then decreases again when the sample is further annealed to the coarse-grained state.

The results of magnetometry presented in this section show, first of all, that nanocrystallinity has a strong impact on the magnetization isotherm of $\mathrm{Gd}$. This becomes particularly clear from the observed dependence of the coercive field and the macroscopic magnetization on the average crystallite size. The variation of $H_{C}$ with temperature essentially reflects the characteristics of the magnetocrystalline anisotropy. An averaging of the anisotropy of the individual grains caused by exchange coupling, as reported in some nanocrystalline soft magnetic materials, ${ }^{50}$ may be present in nanocrystalline $\mathrm{Gd}$ at small grain sizes and temperatures between $200 \mathrm{~K}$ and $T_{C}$, as one can conjecture from the grain-size dependence of coercivity (see Fig. 4). However, the well-known $T_{C}$ shift in nanocrystalline $\mathrm{Gd}$ with grain size may also account for this effect. ${ }^{18}$ The reduction of the macroscopic magnetization in the nanocrystalline material at magnetic fields up to $9 \mathrm{~T}$ and low temperature and, in particular, its variation according to $\Delta M / M \propto D^{-1}$ indicate the special role of the grain boundaries as a dominant source of spin disorder since this scaling law is characteristic for the volume fraction of atoms located in the core regions of grain boundaries. The notion of grain-boundary-induced spin disorder is supported by recent experimental $^{51}$ and theoretical ${ }^{52}$ studies.

The question of how the presence of grain boundaries or other lattice imperfections affects the spin structure of $\mathrm{Gd}$ on a microscopic scale is discussed in the next section in the context of the SANS results. The major advantage of such data is the sensitivity of the SANS method to the magnetic-fielddependent arrangement and spatial extension of perturbations of the magnetization caused by the individual microstructural defects.

\section{Small-angle neutron scattering}

While the nuclear scattering of a texture-free polycrystalline solid only depends on the magnitude and not on the direction of the scattering vector $\mathbf{q}$, the macroscopic scattering cross section $d \Sigma / d \Omega$ of a magnetic material generally contains both isotropic and anisotropic terms [see Eq. (1)]. The twodimensional (2D) detector pattern reflects the superposition of these usually field-dependent contributions. Therefore, as shown in the following section, some characteristics of the underlying spin structure can be inferred immediately from a visual inspection of the $2 \mathrm{D}$ data.

\section{Anisotropy of the SANS pattern}

Figure 5 shows the 2D SANS cross section at $T=5 \mathrm{~K}$ for several applied magnetic field values. At $5 \mathrm{~T}$, intensity maxima perpendicular to the field direction are found [Fig. 5(a)], which is a common result for the SANS of ferromagnetic materials near the saturated state. ${ }^{40,41}$ The distinctiveness of this feature is related to the ratio of magnetic to nuclear scattering, which turns out to be comparatively high in nanocrystalline
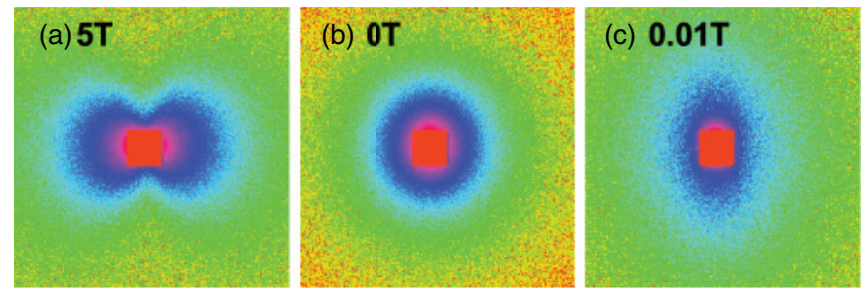

FIG. 5. (Color online) Total SANS cross section of nanocrystalline ${ }^{160} \mathrm{Gd}$ at $T=5 \mathrm{~K}$ and at applied magnetic field values as indicated (logarithmic scale). $\mathbf{H}$ is oriented vertically in the detector plane; pixels in the corners correspond to $q$ values of $1.0 \mathrm{~nm}^{-1}$ in (a) and (b) and $0.2 \mathrm{~nm}^{-1}$ in (c). The central part of the detector with the beam stop has been masked.

${ }^{160} \mathrm{Gd}$, in agreement with the large atomic magnetic moment $\mu_{a}=7.63 \mu_{\mathrm{B}}$, the moderate nuclear scattering length (see Sec. IV C 2), and the low residual porosity of the samples (see Sec. II).

At low fields, the scattering pattern is elongated along the field direction [see Fig. 5(c)]. Such a result is not only less usual than the above, but it also deserves particular attention as this type of anisotropy has been observed previously in the SANS of other nanocrystalline ferromagnets at quite different magnetic fields. ${ }^{41,42,53}$ In zero field, the scattering is essentially isotropic [see Fig. 5(b)]. Note that the anisotropy of the SANS cross section may also depend on $q$, as will be discussed later in this section.

Particularly remarkable results for the 2D SANS cross section are found at intermediate fields. As shown in Fig. 6 for an applied field of $\mu_{0} H=300 \mathrm{mT}$ (measured at $T=78 \mathrm{~K}$ ), a cloverleaf-shaped anisotropy with intensity maxima roughly along the detector diagonals is superimposed on the other scattering contributions. This feature can be seen even more clearly in the difference pattern, when the scattering at the highest field is subtracted. ${ }^{29}$

These results can be understood in terms of the theoretical expression for the SANS cross section of a ferromagnet given by Eq. (1). Comparing the experimental cross section at $5 \mathrm{~T}$ with Eq. (1) shows that the so-called residual scattering $d \Sigma / d \Omega \propto \tilde{N}^{2}+\widetilde{M}_{z}^{2} \sin ^{2} \theta$ dominates in this field range [see Fig. 5(a)]. In particular, the term $\propto \sin ^{2} \theta$ dominates the visible anisotropy, which is related to spatial fluctuations of the magnetization component parallel to the field direction via the Fourier coefficient $\widetilde{M}_{z}$. For low fields, the spin-misalignment scattering becomes more important, and the term $\propto \cos ^{2} \theta$ provides a major contribution to the scattering pattern, which

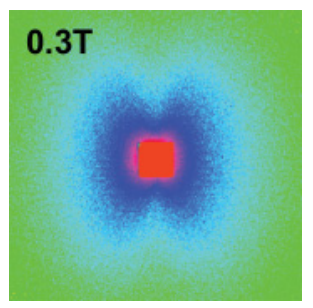

FIG. 6. (Color online) Total SANS cross section (logarithmic scale) at $T=78 \mathrm{~K}$ for an applied magnetic field of $\mu_{0} H=0.3 \mathrm{~T}$ (vertical in the detector plane). Pixels in the corners correspond to a $q$ value of $0.3 \mathrm{~nm}^{-1}$ 

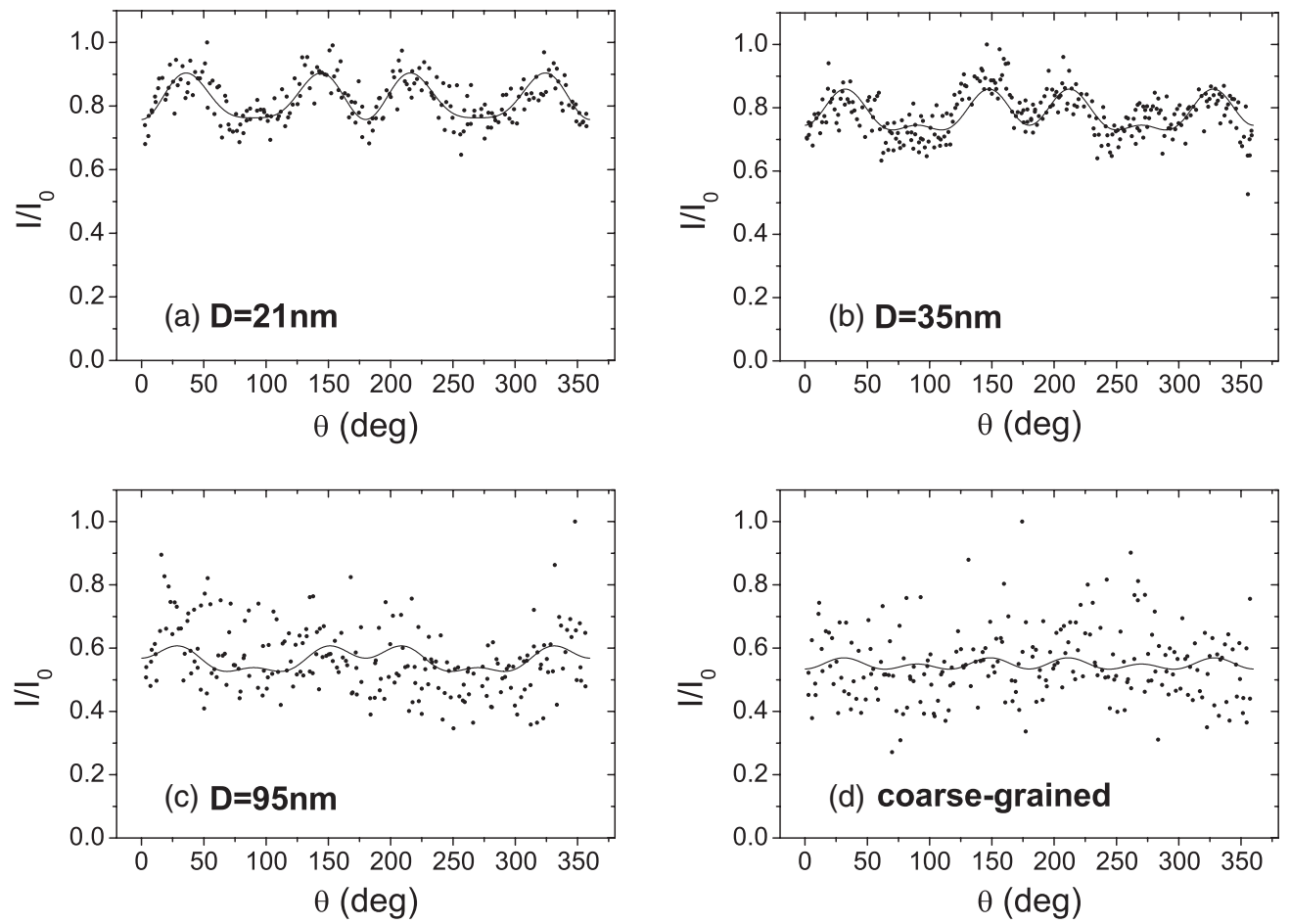

FIG. 7. Normalized total scattering intensity $I / I_{0}$ at $T=78 \mathrm{~K}$ as a function of the angle $\theta$ between the scattering vector and the applied magnetic field $\left(\mu_{0} H=300 \mathrm{mT}\right.$ ), where $I_{0}$ denotes the maximum intensity. $I / I_{0}$ is shown at $q=0.2 \mathrm{~nm}^{-1}$ for grain sizes of (a) $D=21 \mathrm{~nm}$, (b) $D=35 \mathrm{~nm}$, and (c) $D=95 \mathrm{~nm}$, as well as for (d) a coarse-grained reference. Solid lines are a fit to spherical harmonics $Y_{l, 0}(\theta)$ with $l \in\{0,2,4,6\}$.

is associated with the presence of $M_{y}$ fluctuations [see Fig. 5(c)]. A comparable magnitude of $\tilde{M}_{x}$ and $\tilde{M}_{y}$ is expected due to the statistical isotropy of the samples. In zero field, the superposition of all contributions adds up to an essentially isotropic scattering cross section over the displayed $q$ range [see Fig. 5(b)]. Relating the cloverleaf-type scattering contribution observed at intermediate fields to the term $\propto \sin \theta \cos \theta$ seems rather obvious, as this term possesses the appropriate zeros. As discussed below, this conclusion is not entirely straightforward, but nevertheless, the observation of the cloverleaf indicates the presence of a dipole field (e.g., due to a jump in the magnetization ${ }^{43}$ ), which gives rise to correlated fluctuations in $M_{y}$ and $M_{z}$.

In the following it is argued that the cloverleaf pattern for nanocrystalline $\mathrm{Gd}$ can be understood in analogy to the case of the nanocrystalline two-phase alloy Nanoperm, where the jump of the magnetization value between the crystalline Fe particles (volume fraction of $40 \%$ ) and the surrounding amorphous matrix gives rise to a dipolar stray field $\mathbf{H}_{D}$, which was identified as the origin of the unusual cloverleaf-shaped contribution to the scattering cross section. ${ }^{43}$ In particular, the transversal component of the stray field $\mathbf{H}_{D}^{\perp}$ imposes a torque on the magnetization of the surrounding material, thereby giving rise to correlated nanoscale spin disorder. Furthermore, at large applied fields, the response of the magnetization to $H_{D}^{\perp}$ is approximately linear and reflects its angular dependency. The situation in nanocrystalline $\mathrm{Gd}$ appears to be somewhat similar, assuming that strongly localized spin disorder exists in the grain-boundary regions, leading to a reduced effective magnetization, which is in line with the magnetometry data.
The grain-boundary volume fraction of about $20 \%$ may therefore play a role similar to the amorphous matrix of reduced magnetization in Nanoperm, causing the cloverleaf feature in the SANS cross section. Furthermore, due to the low residual porosity of the samples under study (see Sec. II), we do not expect porosity to play a quantitative role for the cloverleaf pattern here. ${ }^{54}$

It should be noted that, according to the above argument, both terms $\left|\tilde{M}_{y}\right|^{2} \cos ^{2} \theta$ and $\tilde{M}_{y} \tilde{M}_{z} \sin \theta \cos \theta$ can be expected to contribute to the cloverleaf, i.e., giving signals $\propto \sin ^{2} \theta \cos ^{4} \theta$ and $\propto \sin ^{2} \theta \cos ^{2} \theta$, respectively. ${ }^{43}$ However, since it is rather difficult to distinguish these two cases based on the experimental data and, in the case of nanocrystalline $\mathrm{Gd}$, not much insight concerning the magnetic microstructure is expected from doing so, we prefer not to discuss this point in more detail.

Subsequent to the above qualitative view on the different anisotropies found in the 2D detector patterns, we focus on a more quantitative evaluation of the data in the following paragraph. This allows us, e.g., to verify the presence of the cloverleaf in the total scattering cross section and to investigate the grain-size dependence of this unusual scattering contribution.

Figure 7 shows the evolution of the cloverleaf anisotropy at $\mu_{0} H=300 \mathrm{mT}$ with increasing crystallite size $D$. The normalized background-corrected total scattering intensity is displayed at $q=0.2 \mathrm{~nm}^{-1}$ for $D=21 \mathrm{~nm}$ [Fig. 7(a)], $D=$ $35 \mathrm{~nm}$ [Fig. 7(b)], $D=95 \mathrm{~nm}$ [Fig. 7(c)], and a coarse-grained reference sample [Fig. 7(d)]. The solid lines are obtained by a fit using spherical harmonics (guides to the eyes). In Figs. 7(a) 
and 7(b) the cloverleaf is clearly visible, with maxima at about $\theta=35^{\circ}, 145^{\circ}, 215^{\circ}$, and $325^{\circ}$. In Fig. 7(b), additional side maxima are found at $90^{\circ}$ and $270^{\circ}$, indicating that the $\sin ^{2} \theta$ contribution is slightly stronger than the $\cos ^{2} \theta$ term (expected maxima at $0^{\circ}$ and $180^{\circ}$ ). However, the nuclear and magnetic scattering that is not related to the cloverleaf adds up to a mainly isotropic background signal in Figs. 7(a) and 7(b). A further increase of the grain size results in an overall isotropic scattering at the displayed values of momentum transfer and magnetic field. Note that the scatter in the data in Figs. 7(c) and 7(d) may be related in part to the normalization but is, in particular, due to the fact that a lower total scattering intensity is detected at a fixed $q$ value when the grain size is increased, which reduces the difference between sample signal and background. However, as the data were recorded with a good overall statistics ( $\approx 10^{6}$ total detector counts), we conclude that the cloverleaf cannot constitute a large scattering contribution at $q=0.2 \mathrm{~nm}^{-1}$ for grain sizes larger than or equal to $95 \mathrm{~nm}$.

Closer inspection of the data at the same temperature and field but at $q=0.07 \mathrm{~nm}^{-1}$ reveals that the cloverleaf is, however, significant at low momentum transfer also for $D=95 \mathrm{~nm}$ (data not shown). Furthermore, the cloverleaf anisotropy vanishes for $D=35 \mathrm{~nm}$ at $q \gtrsim 0.3 \mathrm{~nm}^{-1}$ and for $D=21 \mathrm{~nm}$ slightly above this $q$ value. This suggests that the respective scattering contribution is significant in the $q$ range below the reciprocal grain size, i.e., at $q \lesssim 2 \pi / D$. Consequently, the underlying spin structure appears to be present on a real-space length scale larger than or equal to the average grain size. This result, together with the fact that the cloverleaf was only found in the nanocrystalline state (at all investigated temperatures of $T=5,78$, and $250 \mathrm{~K}$ ), is in line with the above view of strongly localized spin disorder in the core regions of the grain boundaries as possible origin of the cloverleaf in nanocrystalline Gd.

In contrast to the cloverleaf anisotropy, the enhancement of the scattering in the direction of the applied field can be conveniently characterized by the aspect ratio of the 2D SANS pattern. As previously mentioned, this sort of anisotropy is a typical feature of nanocrystalline bulk magnetic materials ${ }^{42,53}$ and may therefore provide useful information regarding the magnetic microstructure and the characteristic length scale of the spin misalignment. Figure 8(a) displays the ratio $I_{P} / I_{S}$ of the background-corrected SANS signal of nanocrystalline Gd parallel and perpendicular to the magnetic-field direction at a fixed value of $q=0.15 \mathrm{~nm}^{-1}$ and $T=78 \mathrm{~K}$, plotted as a function of the applied field for different grain sizes. Above $300 \mathrm{mT}$, the intensity ratio lies between 0.2 and 1.0, while at lower fields values between 1 and 1.4 are found. In this field range, a considerable grain-size dependence is observed, where $I_{P} / I_{S}$ is reduced with increasing grain size and becomes more and more isotropic. At $q>0.5 \mathrm{~nm}^{-1}$, the intensity ratio takes on values $\leqslant 1$ only, which is shown in Fig. 8(b) for the example of $q=1.0 \mathrm{~nm}^{-1}$. Furthermore, no significant grain-size dependence is detected. In Figs. 8(c) and 8(d), the ratio $I_{P} / I_{S}$ is displayed for $D=21 \mathrm{~nm}$ at temperatures of $T=5,78$, and $250 \mathrm{~K}$. A reduction of the intensity ratio with increasing temperature is seen in Fig. 8(c) for $q=0.15 \mathrm{~nm}^{-1}$, in particular, at low and intermediate fields. However, at $q=$ $1.0 \mathrm{~nm}^{-1}$ this is predominantly the case at the highest fields, while at lower fields the scattering is mainly isotropic for all temperatures.

Similar to the previously shown 2D data, these results can be interpreted on the basis of Eq. (1). While at high fields the dominant scattering is expected to be perpendicular to the field direction, the situation changes at intermediate and low fields, and the spin-misalignment scattering becomes important. In particular, the latter is related to terms proportional to $\left|\tilde{M}_{x}\right|^{2}$, $\tilde{M}_{y} \tilde{M}_{z} \sin \theta \cos \theta$ and $\left|\tilde{M}_{y}\right|^{2} \cos ^{2} \theta$. Note that the cloverleaf does not contribute to the data as displayed in Fig. 8. The existence of correlated magnetization fluctuations in the $y$ direction is therefore responsible for the enhanced intensity along $\mathbf{H}$ below $300 \mathrm{mT}$. Such fluctuations are present on a length scale of about $2 \pi / 0.15 \mathrm{~nm}^{-1} \approx 40 \mathrm{~nm}$.

\section{Radially averaged data}

In order to assess the explicit $q$ dependence of experimental SANS data, a radial-averaging procedure is commonly applied. ${ }^{37}$ The respective scattering curves at zero field and $5 \mathrm{~T}$ are shown in Fig. 9 for grain sizes of $D=21, D=35$, and $D=95 \mathrm{~nm}[T=78 \mathrm{~K}$; Fig. 9(a) $]$ and temperature values of $T=5, T=78$, and $T=250 \mathrm{~K}[D=21 \mathrm{~nm}$; Fig. 9(b)]; it is seen that $d \Sigma / d \Omega$ is strongly field dependent at all grain sizes (e.g., a factor of 23 at $D=21 \mathrm{~nm}$ and $\left.q=0.1 \mathrm{~nm}^{-1}\right)$. The point of the strongest field dependence shifts toward lower $q$ with increasing $D$, and the scattering cross section is enhanced in the low- $q$ regime, while a decrease is found at large $q$. The asymptotic exponent $z$ with $d \Sigma / d \Omega \propto q^{-z}$ ranges from $3.25(21 \mathrm{~nm})$ over 3.45 $(35 \mathrm{~nm})$ to $3.65(95 \mathrm{~nm})$ in zero field and from 1.9 over 1.8 to 1.5 at $5 \mathrm{~T}$. Comparing the SANS curves at different temperatures for a grain size of $21 \mathrm{~nm}$ [see Fig. 9(b)], little difference is found between 5 and $78 \mathrm{~K}$. Further temperature increase to $250 \mathrm{~K}$ leads to a reduction of $d \Sigma / d \Omega$ by almost one order of magnitude in zero field. At $5 \mathrm{~T}$ the decrease is less pronounced and takes place predominantly at higher $q$ values.

The observed magnetic field dependence of the SANS cross section provides further strong evidence for the presence of nanoscale spin disorder in nanocrystalline $\mathrm{Gd}$, which can be suppressed by an applied field. The fact that a field dependence is seen up to the highest available momentum transfers indicates that perturbations in the magnetization exist down to a length scale of only a few nanometers, which, as already discussed in the previous section in conjunction with the cloverleaf anisotropy, may be related to microstructural disorder in the vicinity of the grain boundaries.

The radially averaged SANS data at the intermediate fields as shown in Figs. 9(c) and 9(d) reveal the individual magnetic field response of spin disorder on different length scales. It is seen that for both nanocrystalline Gd samples long-range magnetic disorder is suppressed more effectively by an applied field than spin disorder on the shorter length scales. Furthermore, a significant magnetic field effect on the spin structure on the length scale of a few nanometers (i.e., at the largest $q$ ) is observed over the entire field range. This finding is particularly remarkable since basic micromagnetic considerations suggest that short-range spin disorder is essentially suppressed due to the exchange interaction when uniform 

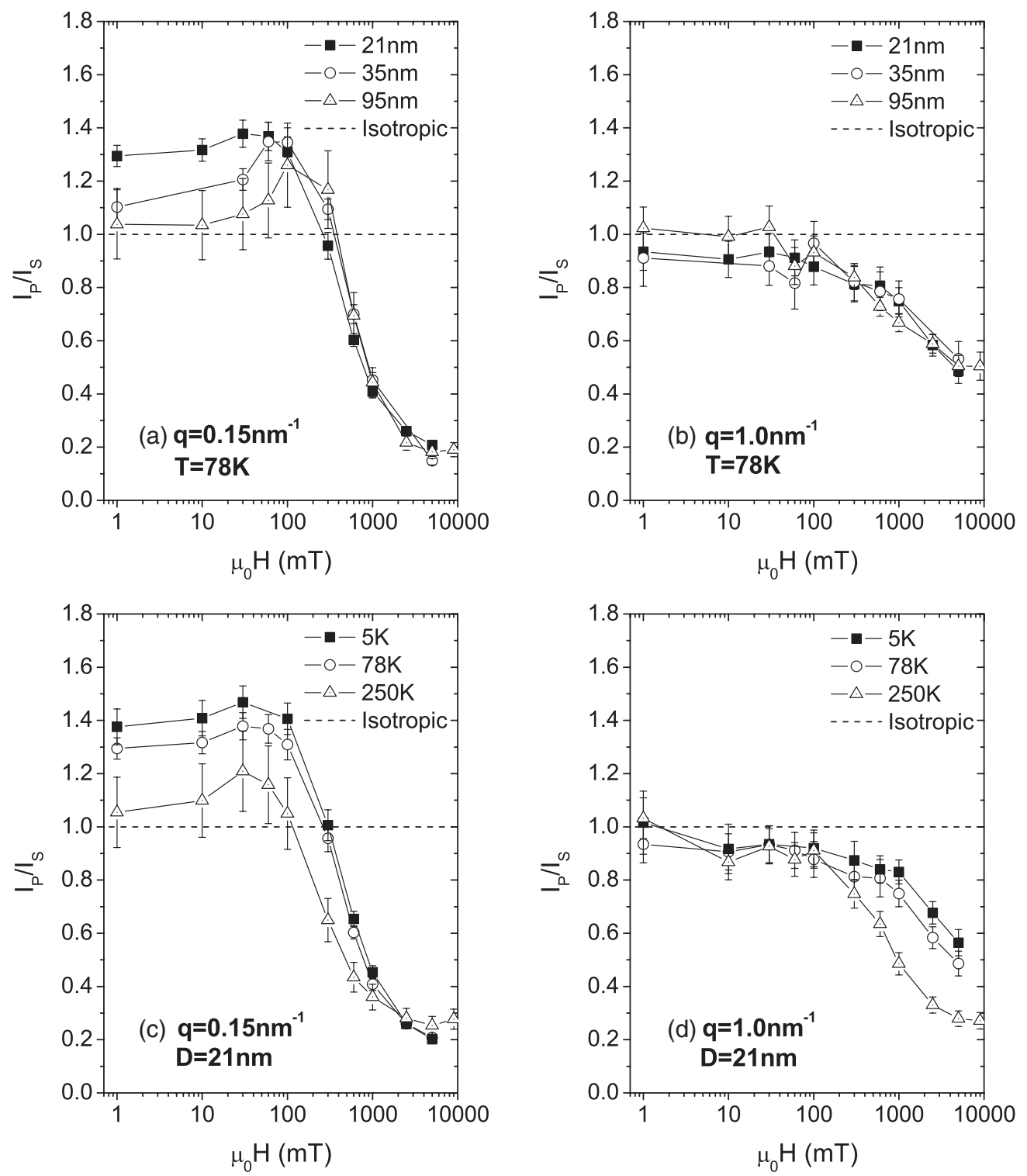

FIG. 8. Applied-field dependence of the ratio $I_{P} / I_{S}$ of the total SANS intensity parallel and perpendicular to $\mathbf{H}$ at various combinations of momentum transfer $q$, grain size $D$, and temperature $T$. Dashed lines show $I_{P} / I_{S}=1$ (isotropic scattering). Solid lines are guides to the eyes.

materials parameters are assumed and the influence of grain boundaries is neglected. ${ }^{41}$

When comparing the magnetic field effect on the scattering curve of nanocrystalline Gd with the results of SANS studies on other nanocrystalline ferromagnets such as $\mathrm{Fe}, \mathrm{Co}, \mathrm{Ni}$, and $\mathrm{Tb},{ }^{41,42,54,55}$ not only the sensitivity of the magnetic microstructure to the applied field but also the ratio of the magnetic and nuclear scattering lengths is of importance. These two quantities have been estimated as $b_{m}=20.6 \mathrm{fm}$ and $b_{n}=(9.12-i 0.18) \mathrm{fm}$, with the latter based on the isotope composition of the present samples. ${ }^{34,56}$ The ratio $b_{m} / b_{n}$ is comparable to the respective values for $\mathrm{Fe}, \mathrm{Co}$, and $\mathrm{Tb},{ }^{34,42,57}$ while Ni possesses a lower relative magnetic scattering length than ${ }^{160} \mathrm{Gd}$. However, the SANS signal of nanocrystalline inert-gas-condensed $\mathrm{Fe}, \mathrm{Co}$, and $\mathrm{Ni}$ was found to depend much less on the applied magnetic field than the data shown in Fig. 9 (see Refs. 54 and 55), while the field effect on nanocrystalline electrodeposited Co and Ni was significantly larger. ${ }^{48}$ These results have been discussed in the context of the significant porosity of inert-gas-condensed $\mathrm{Fe}, \mathrm{Co}$, and $\mathrm{Ni}$ samples, leading to a strong nuclear, i.e., field-independent, scattering contribution, ${ }^{54}$ which was not the case for the electrodeposited samples. Therefore, the strong field effect on $d \Sigma / d \Omega$ that is found for the present ${ }^{160} \mathrm{Gd}$ samples is in line with the low porosity as determined by the Archimedes method (compare Sec. II).

Furthermore, the results on the aforementioned $\mathrm{Fe}, \mathrm{Co}$, and Ni samples do not show any magnetic field response for $q \geqslant$ $0.3 \mathrm{~nm}^{-1}$ (irrespective of the preparation method), in contrast to nanocrystalline $\mathrm{Tb}$, for which a variation of $d \Sigma / d \Omega$ with the magnetic field was still detected above $q=1.0 \mathrm{~nm}^{-1}$ (see Ref. 42). This indicates a general difference regarding the impact of structural defects induced by nanocrystallinity on the magnetic microstructure of rare-earth magnets as compared to 

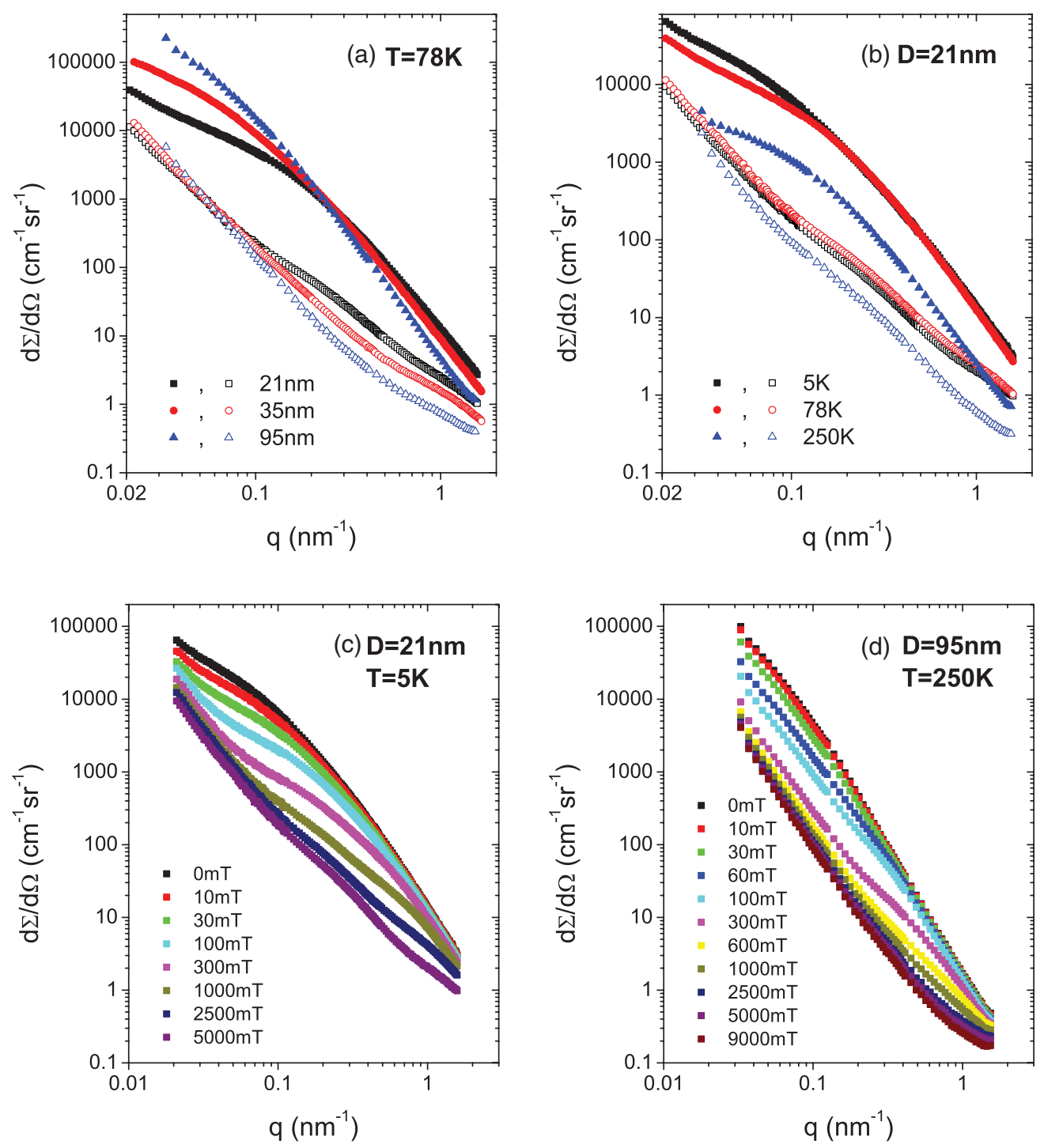

FIG. 9. (Color online) (a) Radially averaged total SANS cross section $d \Sigma / d \Omega$ of Gd at $T=78 \mathrm{~K}$ for different grain sizes $D$ as indicated. (b) $d \Sigma / d \Omega$ for $D=21 \mathrm{~nm}$ and at several temperatures. (c) and (d) Magnetic field dependence of $d \Sigma / d \Omega$ for $D=21$ and $95 \mathrm{~nm}$, respectively, at temperatures as specified. Solid and open symbols in (a) and (b) correspond to field values of 0 and $5 \mathrm{~T}$, respectively.

the $3 d$ transition metals. While in the latter defect-induced perturbations of the magnetization decay on comparatively large length scales, in the former materials strongly localized spin disorder is found.

In accordance with the above view, the asymptotic behavior of the scattering cross section in Fig. 9 does not give an indication for pore scattering, which is usually characterized by a $q^{-4}$ power law at large momentum transfers $q$ (see Refs. 39 and 54). This so-called Porod law is generally expected in small-angle scattering from particle-matrix systems whenever a sharp interface exists between objects embedded in a matrix with a different scattering-length density. Furthermore, in classical small-angle scattering from a scalar scattering-length density distribution $\rho(\mathbf{x})$, exponents with absolute values smaller than 4 are often associated with continuous or fractal transitions between regions of different scattering-length densities. On the other hand, in magnetic SANS from a nanocrystalline random-anisotropy ferromagnet, one may expect exponents with an absolute value larger than 4 , as was observed, e.g., for nanocrystalline $\mathrm{Ni}$ and $\mathrm{Co}^{41}$ It should, however, be noted that the model described in Ref. 41 is based on the assumptions that (i) the magnetocrystalline anisotropy of the individual crystallites is the only source of spin disorder and (ii) the material parameters remain unaltered in the grain-boundary region. Apparently, this is not a good approximation for the case of Gd. Moreover, it may be conjectured that the approach of using micromagnetics theory, which regards the magnetization $\mathbf{M}(\mathbf{x})$ as a continuous function of position $\mathbf{x}$ and assumes only small deviations of the spin orientation from atom to atom, reaches its limits for spin disorder on length scales below a few nanometers (see Refs. 58 and 59). Nevertheless, a quantitative analysis of the radially averaged SANS data is possible based on the correlation function of the spin misalignment. ${ }^{42}$ The respective results are presented in the following section. 

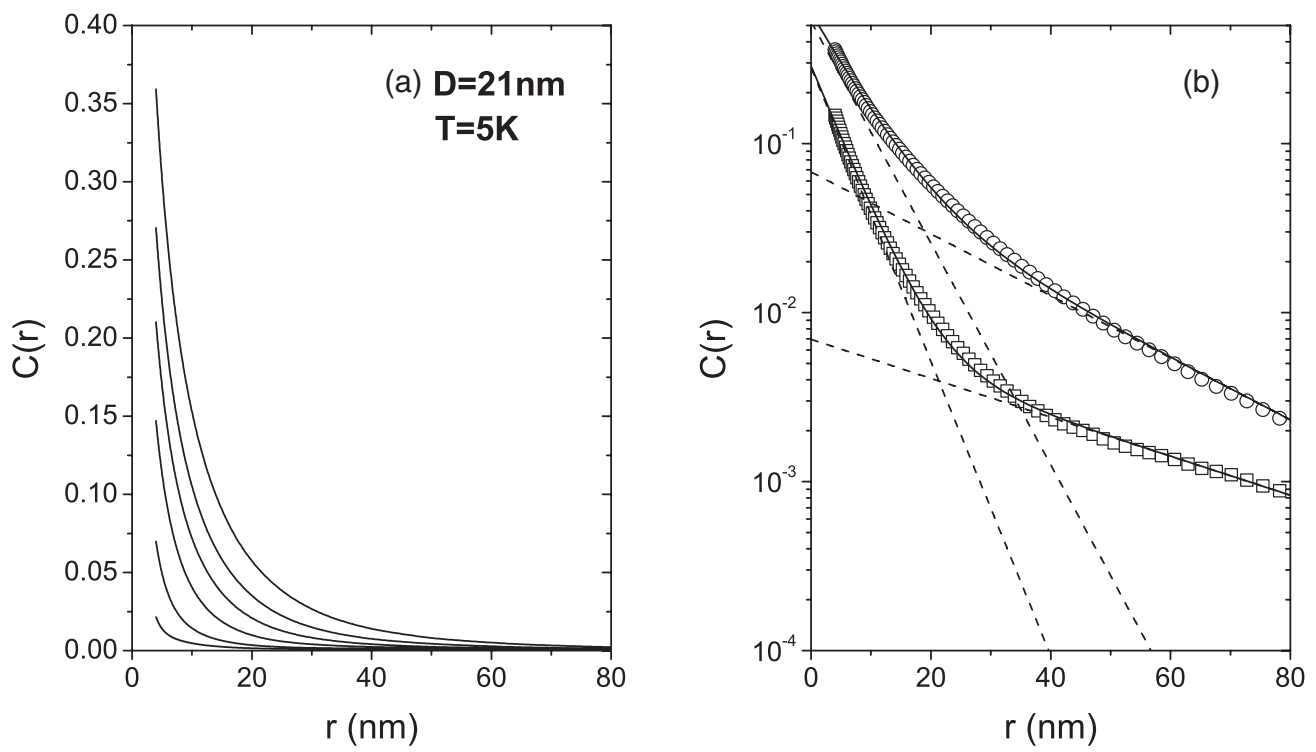

FIG. 10. (a) Correlation function $C(r)$ of the spin misalignment of nanocrystalline $\mathrm{Gd}$ at $T=5 \mathrm{~K}$. Values of the applied magnetic field are, from top to bottom (in mT), 0, 10,30,100,300, and 1000. (b) $C(r)$ from (a) on a log-linear scale at $0 \mathrm{mT}$ (open circles) and $100 \mathrm{mT}$ (open squares). It is seen that the data contain two characteristic lengths scales, as indicated by the dashed lines. Solid lines are a fit to Eq. (4).

\section{Correlation function of the spin misalignment}

The information on the magnetic microstructure of a sample in real space that is contained in experimental SANS data can be accessed in a convenient way by computing the correlation function of the spin misalignment $C(r)$ based on the radially averaged scattering cross section [see Eq. (3)]. As shown in Fig. 10(a) for the case of nanocrystalline $\mathrm{Gd}$, the function $C(r)$ decays monotonically with increasing distance $r$. The numerical value of the correlation function at a given $r$ is reduced with increasing magnetic field, as correlated spin misalignment (with respect to the macroscopic magnetization direction) is progressively suppressed. Two basic parameters characterizing the spin structure are readily extracted, i.e., the correlation length $l_{C}$ and the value $C(0)$ (see Sec. III), where the latter is determined by extrapolation of the $C(r)$ data to $r=0$. In particular, $l_{C}$ is a measure for the distance over which perturbations in the magnetization decay, and $C(0)$ represents the volume-averaged mean-square magnetization fluctuation. ${ }^{42}$

In Fig. 11(a) the magnetic field dependence of $l_{C}$ is shown for different grain sizes $D$. It is seen that at $T=78 \mathrm{~K}$ the correlation length is reduced with increasing field for all grain sizes. In particular at small field values, $l_{C}$ is increased with coarsening of the grain microstructure. All values, ranging from approximately 7 to $17 \mathrm{~nm}$ at zero field and from 4 to $6 \mathrm{~nm}$ at $1 \mathrm{~T}$, are significantly smaller than the respective grain size. The $l_{C}$ results at different temperatures are compared in Fig. 11 (b) for $D=21 \mathrm{~nm}$. Between 5 and $78 \mathrm{~K}$ no major changes are found, while at $250 \mathrm{~K}$, after an initial reduction, the correlation length slightly increases at higher fields.

It is found that the magnetic field dependence of $l_{C}$ at low and intermediate temperatures (with respect to $T_{C}$ ) qualitatively agrees with the micromagnetic perception that local microstructural defects give rise to gradients in the magnetization, which are "transmitted" into the lattice on the nanometer scale via the exchange interaction. ${ }^{60}$ Such static magnetization fluctuations are (with increasing magnetic field) more and more suppressed, as the Zeeman energy contribution to the total energy of the system becomes increasingly important with respect to other terms, such as the exchange energy or the magnetocrystalline anisotropy energy. ${ }^{58}$ For a strongly localized defect, the characteristic range of the perturbation is given by the exchange length of the field, i.e., $l_{C}=l_{H} \propto H_{i}^{-1 / 2}$, where $H_{i}$ denotes the internal field. While this is a reasonable assumption for some types of defects, the magnetocrystalline anisotropy of a material is typically associated with a spatially extended anisotropy field, and in this case the size $\mathcal{L}$ of the defect can be regarded as the average size of regions with a homogeneous anisotropy field. ${ }^{45}$ This view is supported by magnetic-field-dependent SANS data on nanocrystalline $\mathrm{Co}$ and $\mathrm{Ni}$, which showed that the size of the dominating defect is related to the grain size and to the average distance between adjacent stacking faults, respectively; in other words, $\mathcal{L}$ corresponds to the size of crystallographically homogeneous regions in these samples. ${ }^{44}$ In fact, it was found that the field dependence of $l_{C}$ obeys the relation $l_{C}=\mathcal{L}+l_{H}$.

A closer inspection of the $l_{C}(H)$ data in Fig. 11 suggests that the situation in nanocrystalline $\mathrm{Gd}$ is somewhat more complex; e.g., the functional dependence of $l_{C}(H)$ at temperatures of 5 and $78 \mathrm{~K}$ does not yield quantitative agreement with the modified micromagnetic model proposed in Refs. 44 and 45 (not displayed), and at $250 \mathrm{~K}$ an apparent qualitative deviation from the expected behavior is found. Similar results have been observed in nanocrystalline $\mathrm{Tb}$ at $200 \mathrm{~K}$ (see Ref. 42). In this context, the existence of two characteristic length scales was conjectured. As discussed later in this section, the present $C(r)$ data also suggest the existence of two characteristic length scales in the spin structure of nanocrystalline $\mathrm{Gd}$.

The results for the parameter $C(0)$ show a monotonic decrease with increasing field for all data sets (see Fig. 12). In Fig. 12(a) the grain-size dependence of the total mean-square magnetization fluctuation at $T=78 \mathrm{~K}$ is displayed. At low 

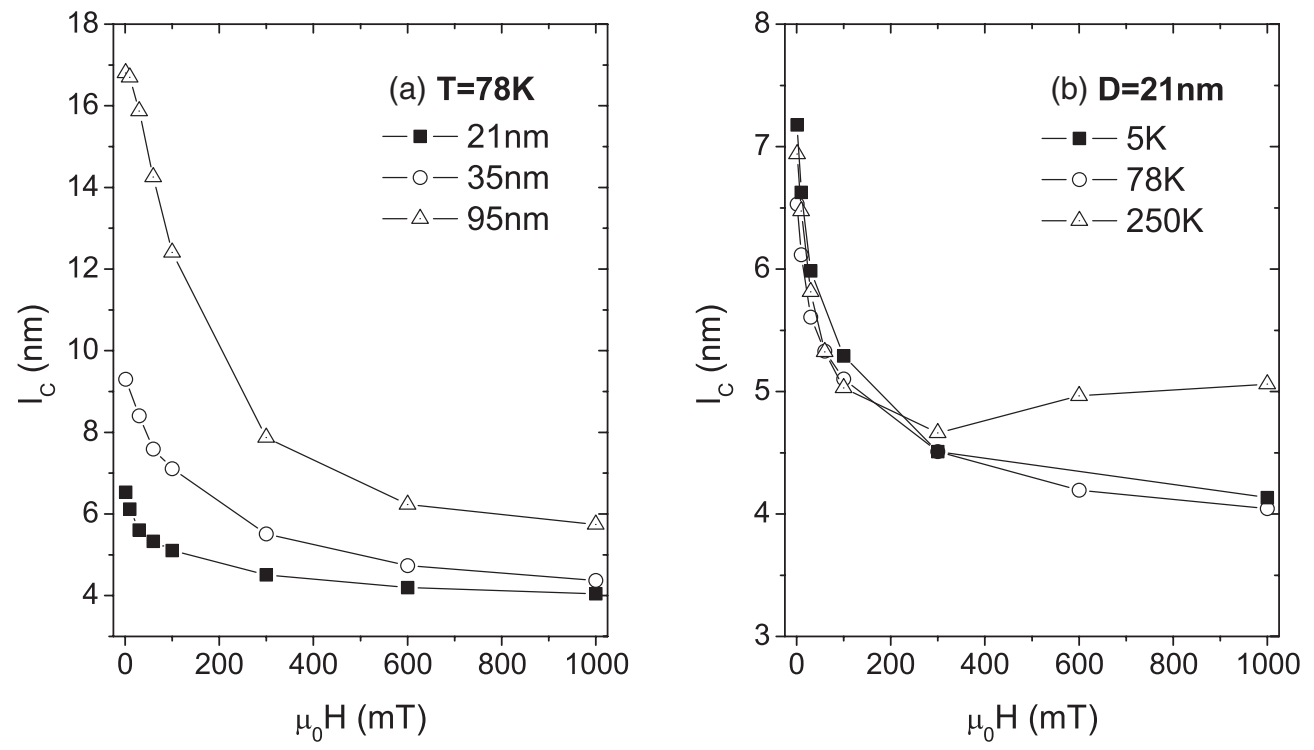

FIG. 11. Correlation length $l_{C}$ of the spin misalignment in nanocrystalline Gd as a function of the applied field $H$ : change of $l_{C}$ (a) with grain size at $T=78 \mathrm{~K}$ and (b) with temperature at $D=21 \mathrm{~nm}$. Lines are guides to the eyes.

fields $C(0)$ is slightly enhanced with increasing grain size, while apparently at higher fields this trend is reversed. The data measured at different temperatures show little change between $T=5$ and $78 \mathrm{~K}$ [see Fig. 12(b)], while considerably reduced values are obtained at $T=250 \mathrm{~K}$. Note that the calculation of the correlation function via Eq. (3) involves the magnetic scattering length $b_{m}$, which contains the temperature-independent value of the atomic magnetic moment $\mu_{a}=7.63 \mu_{\mathrm{B}}$ of $\mathrm{Gd}$. By contrast, the SANS cross section of a ferromagnet depends on the (temperature-dependent) magnetization. Therefore, the $C(0)$ values have been scaled with the factor $\left[M_{S}(0) / M_{S}(T)\right]^{2}$ in Fig. 12 in order to facilitate the comparison of the temperature-dependent results for the total correlated spin misalignment.
Figure 13 displays the values of the macroscopic magnetization of nanocrystalline $\mathrm{Gd}$ at $T=5 \mathrm{~K}$ calculated from $C(0)$, together with data obtained by classical magnetometry, for grain sizes as indicated. It is seen that the values derived from SANS are considerably larger than the directly measured volume-averaged magnetization $|\langle\mathbf{M}\rangle|$ over the whole field range, despite the slightly smaller grain size. Since the quantity $C(0)$ is identical to the mean-square deviation of $\mathbf{M}$ from the macroscopic average magnetization, this discrepancy can contribute to an understanding of the nature of the spin disorder in nanocrystalline Gd.

When comparing the two "magnetization curves" in Fig. 13, it is important to keep in mind the experimental resolution of the SANS technique. In particular, the real-space range of
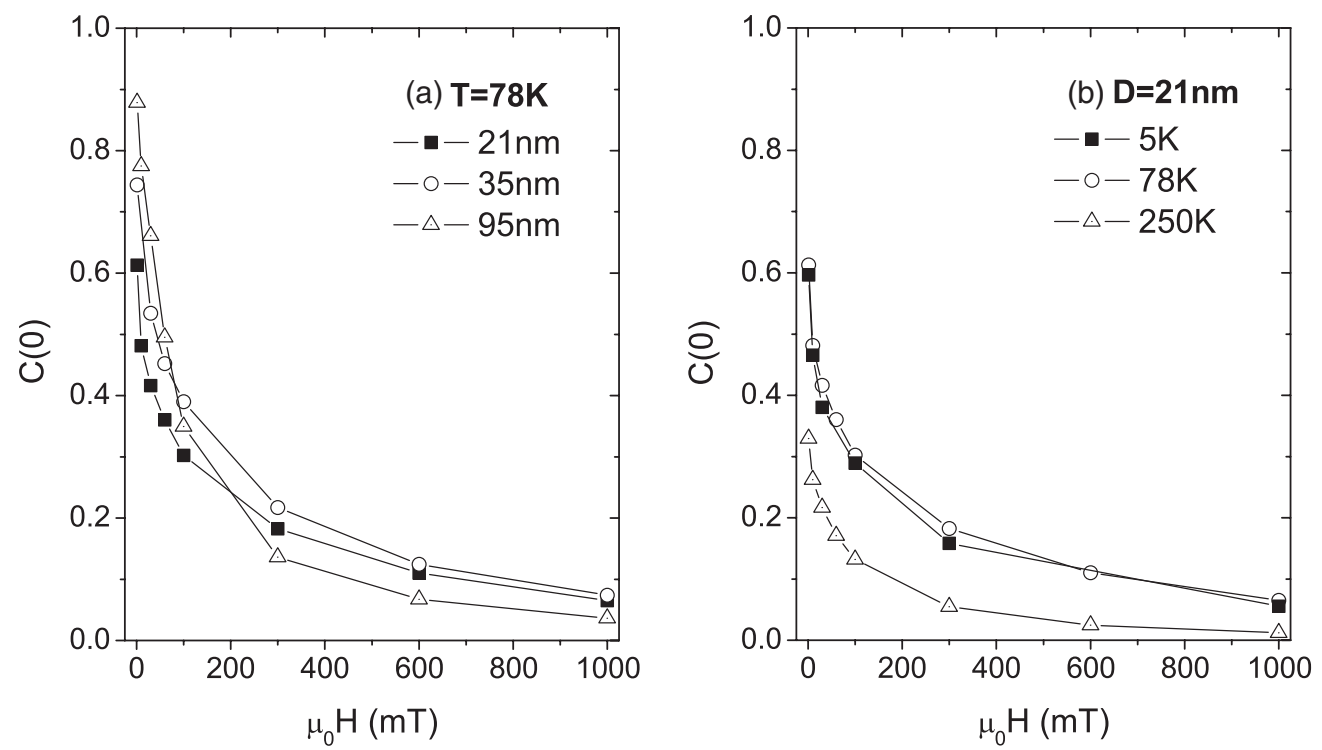

FIG. 12. Extrapolated values $C(0)$ of the correlation function $C(r)$ for $r \rightarrow 0$ as a function of $H$ at (a) fixed temperature $T=78 \mathrm{~K}$ for variable grain size $D$ and (b) at fixed grain size $D=21 \mathrm{~nm}$ and temperatures as indicated. Lines are guides to the eyes. 


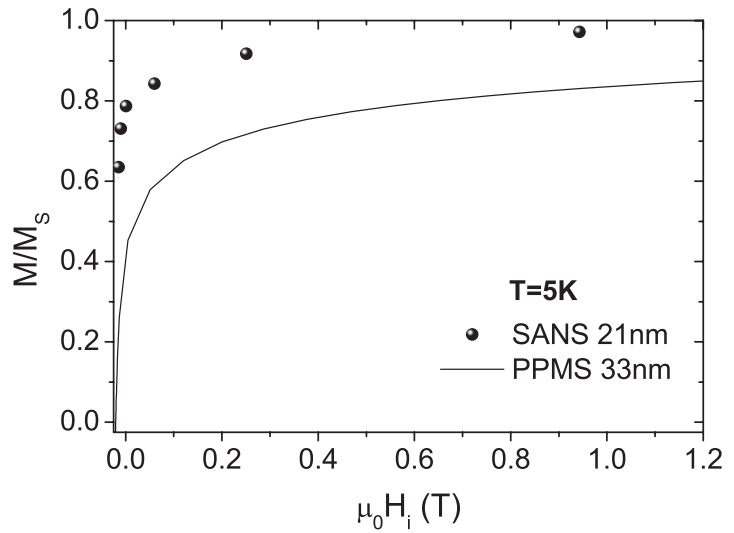

FIG. 13. Normalized magnetization $M / M_{S}$ of nanocrystalline $\mathrm{Gd}$ at $T=5 \mathrm{~K}$ as a function of the internal field $H_{i}$ (using $\mu_{0} M_{S}=2.69 \mathrm{~T}$ from Ref. 6). The solid line shows PPMS data for a grain size of $D=33 \mathrm{~nm}$. Dots show magnetization values calculated from the correlation function $(D=21 \mathrm{~nm})$ according to $M / M_{S}=\sqrt{1-C(0)}$ (see Ref. 42).

static spin fluctuations that is probed in our SANS experiment is limited by approximately $\left[2 \pi q_{\max }^{-1}-2 \pi q_{\min }^{-1}\right]$ (i.e., $4-200$ $\mathrm{nm}$ in the present setup). Therefore, a significant fraction of the total spin misalignment may not be reflected in the SANS data. While long-range magnetization fluctuations, at least at the higher fields, are not likely to contribute much to the total magnetization reduction, it seems plausible that spin disorder on a length scale below $4 \mathrm{~nm}$ accounts for a major part of the reduction of the macroscopic magnetization in this material, in accordance with the considerable field dependence of the radially averaged scattering cross section at the highest $q$ and the highest fields (see Sec. IV C 2).

Possible reasons for the observed reduction of $C(0)$ at $T=250 \mathrm{~K}$ are the reduction of $T_{C}$ in the nanocrystalline

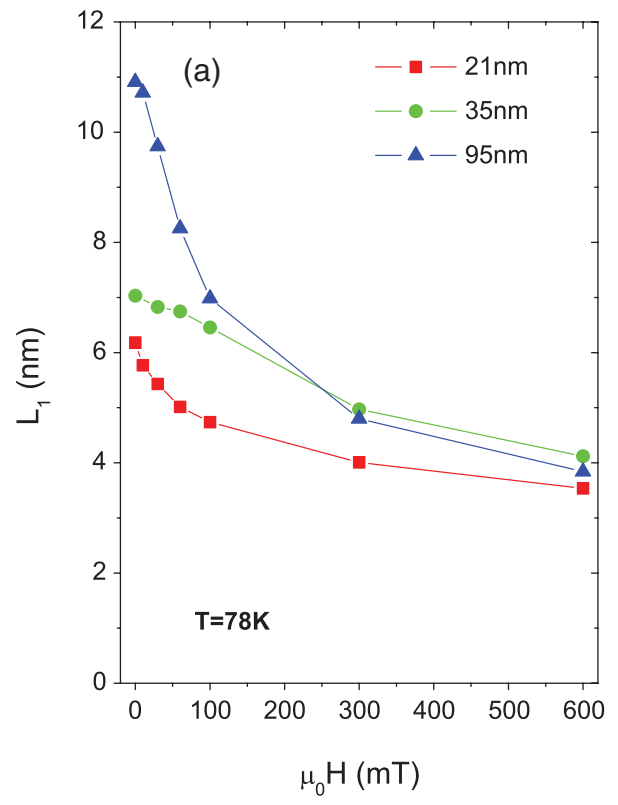

state, ${ }^{18}$ the temperature dependence of the magnetocrystalline anisotropy, ${ }^{5}$ or a combination thereof. The grain-size dependence of $C(0)$ is discussed in the following paragraphs.

Although the above data analysis already allows one to estimate the average range and strength of nanoscale perturbations of the magnetization, the question needs to be addressed as to whether these are indeed caused by more than one type of defect, as previously discussed, and if this is the case, what is the range and strength of the perturbations caused by the individual defects. As can be seen in Fig. 10(b), a closer inspection of the correlation function on a semilogarithmic scale implies the presence of two characteristic length scales of the spin misalignment in nanocrystalline $\mathrm{Gd}$ with a grain size of $D=21 \mathrm{~nm}$ (see also Fig. 1 in Ref. 30). It also becomes clear that the above-mentioned value of $l_{C}$ contains both contributions with individual weights. In order to extract the two correlation lengths $L_{1}$ and $L_{2}$ we have performed a fit of the $C(r)$ data to the sum of two exponentials,

$$
C(r)=C_{1} \exp \left(-r / L_{1}\right)+C_{2} \exp \left(-r / L_{2}\right),
$$

subject to the constraint $C_{1}+C_{2}=C(0)$. Here, $C(0)$ again denotes the total mean-square magnetization fluctuation determined by extrapolation, as described above. Note that choosing the exponential, although reasonable from micromagnetics theory, ${ }^{60}$ is somewhat arbitrary and merely provides a selfconsistent approach to access the desired parameters. However, it is found that Eq. (4) allows an excellent quantitative description of the data. In the following, the recent grain-sizedependent results are presented together with those previously reported on the nanocrystalline $(D=21 \mathrm{~nm})$ as-prepared ${ }^{160} \mathrm{Gd}$ sample. ${ }^{30}$

Figure 14 displays the magnetic-field dependence of the parameters $L_{1}$ and $L_{2}$ at $T=78 \mathrm{~K}$ for three different grain sizes. It is seen that the first correlation length $L_{1}$ is always

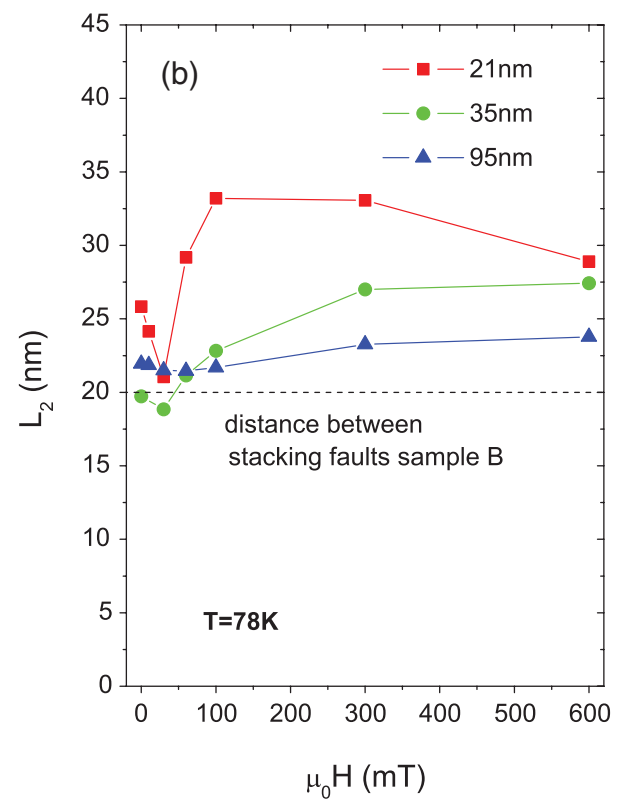

FIG. 14. (Color online) Applied-field dependence of the correlation lengths (a) $L_{1}$ and (b) $L_{2}$ for grain sizes as indicated in the legends. $L_{1}$ and $L_{2}$ were obtained from a fit of the correlation function $C(r)$ using Eq. (4). Solid lines are guides to the eyes. The dashed line in (b) is the average distance between stacking faults. 

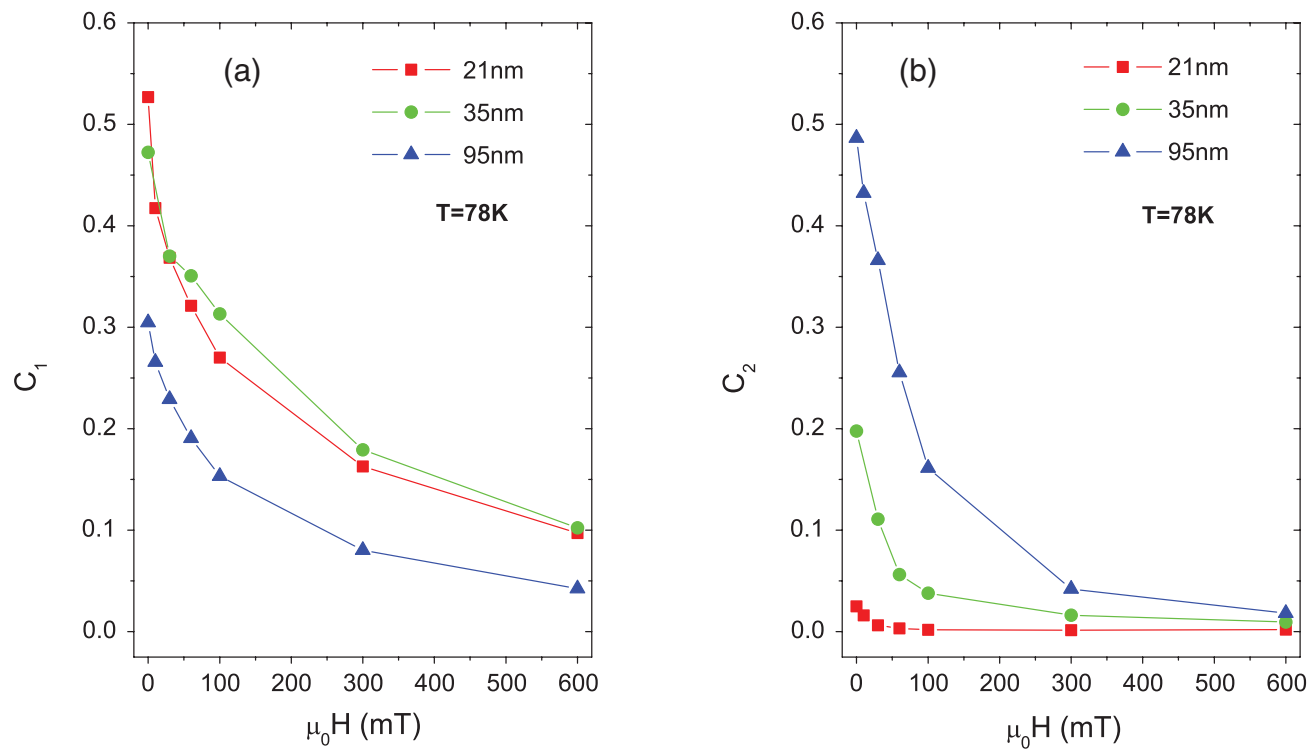

FIG. 15. (Color online) Magnetic field dependence of the parameters (a) $C_{1}$ and (b) $C_{2}$ at $T=78 \mathrm{~K}$ for grain sizes as indicated. In the fit of the model function [see Eq. (4)] to the $C(r)$ data, $C_{1}$ and $C_{2}$ represent the respective weights associated with the correlation lengths $L_{1}$ and $L_{2}$. The two parameters are related by the condition $C_{1}+C_{2}=C(0)$. Lines are guides to the eyes.

smaller than $L_{2}$ and decreases monotonically with increasing field for all grain sizes. The numerical values in zero field are, respectively, 6,7 , and $11 \mathrm{~nm}$, whereas at $600 \mathrm{mT}$ all $L_{1}$ values are equal to $4 \mathrm{~nm}$ within the experimental uncertainty of \pm 1 $\mathrm{nm}$. The results for $L_{2}$ do not show a clear trend. Taking into account the experimental error of $\pm 5 \mathrm{~nm}$, however, a minor increase with the applied magnetic field is seen, and for larger grain sizes this correlation length is slightly reduced. Overall, $L_{2}$ values between 20 and $35 \mathrm{~nm}$ are found.

The results for the respective weights $C_{1}$ and $C_{2}$ displayed in Fig. 15 show a reduction of magnetization fluctuations on both length scales with increasing field. Furthermore, it is worth noting that with increasing grain size spin misalignment with the characteristic length $L_{2}$ gains importance relative to fluctuations on the length scale $L_{1}$, coupled to the weight $C_{1}$. This is particularly relevant at the smaller fields, and the increase of the total mean-square magnetization fluctuation $C(0)$ with grain size found in that field range (see Fig. 12) can be attributed to the contribution $C_{2}$. The errors in $C_{1}$ and $C_{2}$ were estimated as \pm 0.04 and \pm 0.006 , respectively. Note that, in accordance with Ref. 30, the data displayed in Fig. 15 have not been scaled to $M_{S}(T)$. The results at 21-nm grain size and $5 \mathrm{~K}$ are very similar to those at $78 \mathrm{~K}$, apart from a slight increase in $L_{2}$ and $C_{2}$. At $250 \mathrm{~K}$ a nonvanishing value for $C_{2}$ was only found above $300 \mathrm{mT}$, in agreement with the observed increase of the overall correlation length $l_{C}$ [see Fig. 11(b)]. The quantities $L_{1}$ and $L_{2}$ take on values of 4 and 20-35 nm at this temperature, respectively.

Neither $L_{1}$ nor $L_{2}$ can be described by means of a micromagnetic model function as proposed in Refs. 44 and 45. In fact, basic micromagnetic models may not be adequate for the description of the spin structure of nanocrystalline $\mathrm{Gd}$ since local variations of the spin orientation on a length scale of about ten times the interatomic distance ${ }^{61}$ or below reach the limit of a continuous magnetization vector field description. ${ }^{59}$ Furthermore, a deviation from the behavior described in Refs. 44 and 45 is not surprising since strong internal magnetostatic stray fields are generally coupled to such an inhomogeneous nanoscale magnetic microstructure. ${ }^{62}$

In accordance with the previous results and in view of the measured numerical values it seems plausible to relate the characteristic lengths $L_{1}$ and $L_{2}$ with the two basic microstructural defects present in nanocrystalline solids, namely, the atomic-site disorder associated with the grain boundaries and the variation of the crystallographic orientation on the length scale of the grain size, where the latter is associated with a statistic orientation of the magnetocrystalline anisotropy ("random anisotropy"). In the case of the grain boundaries, this notion is immediately consistent with the data. In particular, the asymptotic value of $L_{1}=4 \mathrm{~nm}$, which is independent of the grain size, and the reduction of the contribution $C_{1}$ to the total spin misalignment with larger crystallite size are in excellent agreement with this view. The finding that $C_{2}$ decays much faster than $C_{1}$ upon application of a magnetic field and that the latter takes on nonvanishing values for the higher fields is consistent with the results from magnetometry shown in Sec. IV B, which also suggest that grain-boundaryinduced spin disorder in nanocrystalline Gd allows only partial saturation of this material at field values typically available in a laboratory.

The results for $L_{2}$, however, do not seem to be in line with the above picture at first glance. In fact, the values of the second correlation length of $20-35 \mathrm{~nm}$ measured for the annealed Gd sample are considerably smaller than one can expect for crystallite sizes of 35 and $95 \mathrm{~nm}$ since, according to the above-mentioned relation $l_{C}=l_{H}+\mathcal{L}$, values larger than or equal to the average crystallite size should be found. However, the situation appears to be similar to that in nanocrystalline $\mathrm{Ni}$, where a correlation length smaller than the crystallite size was measured, which could be related to the distance between neighboring stacking faults. ${ }^{48}$ The stacking-fault density present in the nanocrystalline ${ }^{160} \mathrm{Gd}$ samples used for SANS 
has been estimated via XRD (for details see Sec. IV A and Appendix B). A significant growth-fault density $\beta$ was found in sample $B$, which was stepwise annealed. The numerical values are $\beta=3.7 \%$ in the as-prepared state (no SANS data available), $1.9 \%(D=35 \mathrm{~nm})$, and $1.2 \%(D=95 \mathrm{~nm})$. The respective average distances between neighboring stacking faults of 15 and $24 \mathrm{~nm}$ show reasonable agreement with the characteristic length $L_{2}$. In sample A with an as-prepared grain size of $21 \mathrm{~nm}$, no stacking faults were found within the experimental uncertainty. The results for $L_{2}$ may therefore be seen as an indication that in Gd the perturbation of the magnetization due to the magnetocrystalline anisotropy field is limited to the range between adjacent stacking faults. Furthermore, an additional contribution from magnetoelastic coupling to the total magnetic anisotropy present in a crystalline subdomain may be conjectured due to the considerable microstrain $\epsilon$ found in the samples. ${ }^{19,63,64}$

\section{SUMMARY AND CONCLUSIONS}

We have investigated nanocrystalline Gd samples of several grain sizes by means of magnetometry and magnetic smallangle neutron scattering, where the latter has been facilitated by the use of the low-capturing isotope ${ }^{160} \mathrm{Gd}$. As a result of our study, the grain boundaries have been identified as the dominant source of spin disorder in nanocrystalline $\mathrm{Gd}$. The observed difference between the magnetometry data and the magnetization curve calculated from the SANS cross section (Fig. 13) suggests strongly localized magnetic disorder on the atomic scale, a finding that is supported by the grainsize dependence of the high-field magnetization curves, the reduction of $M$ being proportional to $D^{-1}$ (Fig. 3). The magnetostatic stray field that is associated with the spin disorder due to the grain-boundary component provides an explanation for the existence of the cloverleaf anisotropy that is observed in the SANS pattern at intermediate fields. Our results indicate that, in the immediate vicinity of the grain boundaries, correlated spin misorientation on a characteristic length scale $L_{1} \sim 4-10 \mathrm{~nm}$ exists. Furthermore, a second correlation length $L_{2} \sim 20-35 \mathrm{~nm}$ was found, characterizing spin misalignment that is caused by the magnetocrystalline anisotropy within the individual crystallites (or coherently ordered crystalline regions), giving rise to an anisotropy of the $\cos ^{2} \theta$ type in the SANS cross section. In particular, this becomes relevant at applied fields below $300 \mathrm{mT}$. At low fields and for grain sizes larger than $35 \mathrm{~nm}$ the magnetocrystalline anisotropy accounts for the major part of the correlated spin disorder in nanocrystalline $\mathrm{Gd}$.

\section{ACKNOWLEDGMENTS}

We thank Adrian Ferdinand, Mihdi Elmas, and Kristian Döbrich for assistance during the SANS experiments and Jürgen Markmann for fruitful discussions regarding the XRD data analysis. This study was financially supported by the Universität des Saarlandes, by the European Commission (Contract No. RII-CT-2003-505925), by the Deutsche Forschungsgemeinschaft (Grants No. MI 738/3-2 and No. MI 738/6-1), and by the National Research Fund of Luxembourg in the framework of ATTRACT Project No. FNR/A09/01. Part of this work is based on experiments performed at the Swiss spallation neutron source SINQ, Paul Scherrer Institute, Villigen, Switzerland.

\section{APPENDIX A: ESTIMATION OF THE CONTRIBUTION OF A LARGE VOLUME FRACTION OF GRAIN BOUNDARIES TO THE MAGNETIZATION OF A POLYCRYSTALLINE FERROMAGNET}

In this section we provide an estimate for the relative reduction of the magnetization $\Delta M / M:=\left(M_{\mathrm{cg}}-M_{\mathrm{nc}}\right) / M_{\mathrm{cg}}$ of a polycrystal as a function of the average crystallite size $D$. In particular, we consider the case of a lowered magnetization $M_{\mathrm{GB}}$ within the grain boundaries, as compared to the bulk of the grains. ${ }^{65}$ The quantities $M_{\mathrm{cg}}$ and $M_{\mathrm{nc}}$ represent, respectively, the volume-averaged magnetizations of the coarse-grained and the nanocrystalline material. We start out by assuming that the total magnetic moment $m$ of the saturated sample is composed of a contribution $m_{\mathrm{X}}$ due to the bulk of the crystallites and of a part $m_{\mathrm{GB}}$ due to the grain-boundary component, i.e., $m=m_{\mathrm{X}}+m_{\mathrm{GB}}$. The vector character of the magnetic moment is not considered here, as we focus on the saturated state. ${ }^{66}$ The total volume $V$ is written as the sum of $V_{\mathrm{X}}$ and $V_{\mathrm{GB}}$, where the volume of the grain-boundary "phase" is expressed as $V_{\mathrm{GB}}=A_{\mathrm{GB}} \delta$, with $A_{\mathrm{GB}}$ being the total interface area and $\delta$ denoting an effective grain-boundary thickness. By using the stereological identity $A_{\mathrm{GB}} / V=2 / L_{A},{ }^{49,67}$ where $L_{A}$ denotes the area-weighted mean column length of the crystallites, we obtain for the grain-size-dependent magnetization

$$
\frac{m}{V}=M_{\mathrm{X}}\left(1-\frac{2 \delta}{L_{A}}\right)+M_{\mathrm{GB}} \frac{2 \delta}{L_{A}} .
$$

By identifying the grain-size-independent magnetization $M_{\mathrm{cg}}$ with $M_{\mathrm{X}}=m_{\mathrm{X}} / V_{\mathrm{X}}$ and $M_{\mathrm{nc}}$ with Eq. (A1), the final expression for $\Delta M / M$ reads

$$
\Delta M / M=\frac{2 \delta}{L_{A}}\left(1-\frac{M_{\mathrm{GB}}}{M_{\mathrm{X}}}\right) .
$$

Both expressions yield the expected result for a vanishing volume fraction of interfaces, i.e., for $L_{A} \rightarrow \infty$. Note also that the influence of triple lines and quadruple-point junctions has been neglected. This seems permissible since both defects are expected to be relevant in nanocrystalline materials for $L_{A} \lesssim$ $5 \mathrm{~nm} .{ }^{68}$ Furthermore, we do not take into account the effect of grain-boundary excess volume since a constant volume is used. ${ }^{69}$ However, it is readily verified by a similar derivation that the expressions obtained here are equally valid for the mass magnetization (i.e., the magnetic moment of a sample normalized to its mass). In particular, since the mass of a sample is invariant with respect to the grain size, changes in density, e.g., due to changes in grain-boundary excess volume, do not contribute to the mass magnetization.

By further assuming that the shape of the crystallites is spherical and that their sizes are lognormally distributed with a typical width of $\sigma=1.7$ (see Ref. 67), we can express $L_{A}=$ $2 / 3 \exp \left(-\ln \sigma^{2}\right) D \cong 0.5 D$ in terms of the (volume-averaged) mean grain size $D$, which is obtained from the analysis of wideangle $\mathrm{x}$-ray diffraction data. The ratio of the grain boundary to 
bulk magnetization is then obtained as

$$
\frac{M_{\mathrm{GB}}}{M_{\mathrm{X}}}=1-\frac{\Delta M}{M} \frac{D}{4 \delta} .
$$

Using $D=21 \mathrm{~nm}, \delta=1 \mathrm{~nm}$, and $\Delta M / M=5 \%$, we obtain a volume fraction of grain boundaries of roughly $20 \%$, and the relative reduction of the grain-boundary magnetization $M_{\mathrm{GB}}$ with respect to the bulk of the grains amounts to $26 \%$.

\section{APPENDIX B: ESTIMATING THE STACKING-FAULT DENSITY OF NANOCRYSTALLINE HCP MATERIALS FROM XRD USING FWHM DATA}

In a hexagonal-closed-packed (hcp) lattice two different types of stacking faults are generally distinguished, which may be referred to as deformation and growth faults. ${ }^{46,70}$ In the following, the respective stacking-fault densities are denoted as $\alpha$ and $\beta$.

In contrast to the fcc crystal, the diffraction pattern of a hcp structure does not show relative peak shifts due to stacking faults. Furthermore, the analysis of general size broadening (including the one caused by stacking faults) based on the Warren-Averbach method, which is, in principle, applicable to hep diffraction data, ${ }^{71}$ requires a high accuracy in the foot region of the peaks and is thus complicated by the strong peak overlap in nanocrystalline hcp materials. Therefore, we have chosen an alternative approach via the conveniently accessible FWHM data.

The hcp peak broadening induced by stacking faults $B_{2 \theta}^{0}$ can be related to the Miller indices $(h k l)$ of the respective lattice planes by

$$
\begin{aligned}
& B_{2 \theta}^{0}=\left(360 / \pi^{2}\right) \tan \theta|l|(d / c)^{2}(3 \alpha+3 \beta), \\
& B_{2 \theta}^{0}=\left(360 / \pi^{2}\right) \tan \theta|l|(d / c)^{2}(3 \alpha+\beta),
\end{aligned}
$$

for even and odd $l$, respectively, if the condition $h-k=3 N \pm$ 1 is satisfied, where $N \in\{0,1,2, \ldots\}$ (see Ref. 46). In all other cases, the respective peak is not affected by stacking faults. In Eqs. (B1) and (B2), the quantities $2 \theta, d$, and $c$ denote the scattering angle, the interplanar spacing, and the hcp lattice parameter in the $c$ direction, respectively. The value of $d$ can, e.g., be obtained by determination of the peak positions and subsequent use of the Bragg equation.

A major obstacle for the direct use of Eqs. (B1) and (B2) on XRD data from nanocrystalline materials is the fact that, usually, a large fraction of the peak broadening is caused by the small crystallite size $D$ and in most cases also by a large degree of inhomogeneous microstrain $\epsilon$. However, the respective contributions can be separated, as is illustrated in the following.

For this purpose, the two parameters $D$ and $\epsilon$ have been determined by the method of Klug and Alexander, ${ }^{33}$ using the

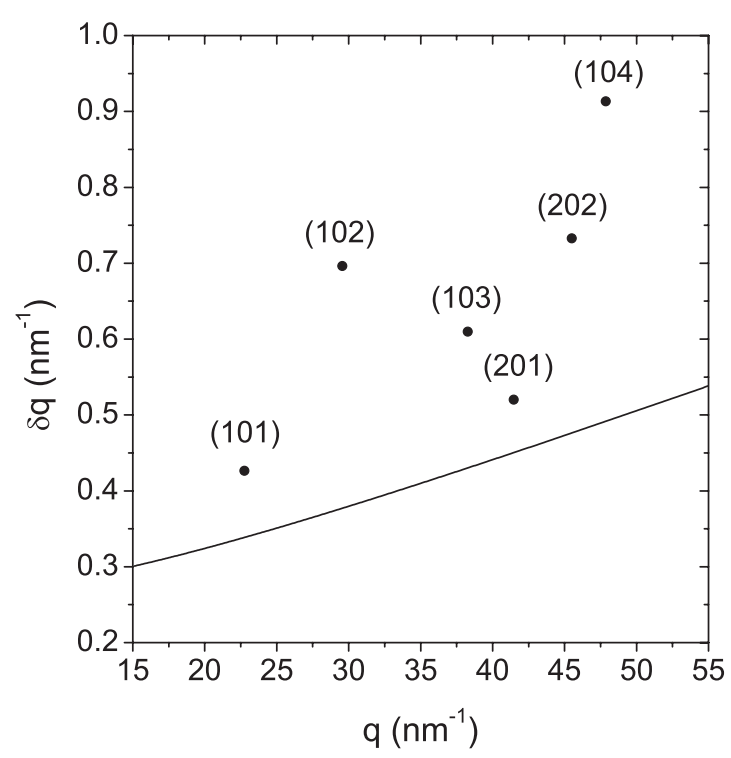

FIG. 16. The dots represent the total broadening of the peaks affected by stacking faults for nanocrystalline ${ }^{160} \mathrm{Gd}$ (sample B, as prepared). The solid line shows the calculated peak broadening using Eq. (B3) based on crystallite size and microstrain only; the employed values $D=25.4 \mathrm{~nm}$ and $\epsilon=0.349 \%$ were calculated from the FWHM data of the peaks that are not affected by stacking faults. The difference between the displayed data points and Eq. (B3) is seen to agree with the predictions of Eqs. (B1) and (B2) regarding both sign and absolute value.

experimental data of the peaks that are not affected by stacking faults, i.e., (100), (002), (110), (200), (112), and (004). Based on the values for $D$ and $\epsilon$ obtained in this way, the pure broadening due to $D$ and $\epsilon$ for the peaks affected by stacking faults, i.e., (101), (102), (103), (201), (202), and (104), can be calculated in reciprocal space via

$$
\delta q=\frac{4 \pi K}{3 D}+\sqrt{\left(\frac{4 \pi K}{3 D}\right)^{2}+4 \epsilon^{2} q^{2}},
$$

where $q=4 \pi \sin \theta / \lambda$ denotes the scattering vector and $K=0.83$ is the Scherrer constant for FWHM data. ${ }^{32}$ The computed values $\delta q$ can be subtracted (assuming a Lorentzian peak profile) from the measured widths of the respective peaks, which have been previously corrected for instrumental broadening (see Fig. 16). Note that this approach involves an approximation insofar as the contribution of microstrain to the peak shape is assumed to be Gaussian in the Klug and Alexander analysis.

From the present XRD data on $\mathrm{Gd}$ three independent values for each of the factors $3 \alpha+3 \beta$ and $3 \alpha+\beta$ in Eqs. (B1) and (B2) were extracted, providing an overdetermined set of equations for the stacking-fault densities $\alpha$ and $\beta$. The results are summarized in Table I.

\footnotetext{
*f.doebrich@witchhunt.de

${ }^{\dagger}$ Present address: European Spallation Source ESS AB, Box 176, S-22100 Lund, Sweden.
}

${ }^{1}$ R. P. Cowburn, J. Phys. D 33, R1 (2000).

${ }^{2}$ Advanced Magnetic Nanostructures, edited by D. Sellmyer and R. Skomski ( Springer, New York, 2006). 
${ }^{3}$ F. Kronast, N. Friedenberger, K. Ollefs, S. Gliga, L. Tati-Bismaths, R. Thies, A. Ney, R. Weber, C. Hassel, F. M. Römer, A. V. Trunova, C. Wirtz, R. Hertel, H. A. Dürr, and M. Farle, Nano Lett. 11, 1710 (2011).

${ }^{4}$ J. Jensen and A. R. Mackintosh, Rare Earth Magnetism (Clarendon, Oxford, 1991).

${ }^{5}$ C. D. Graham Jr., J. Appl. Phys. 34, 1341 (1963).

${ }^{6} \mathrm{~S}$. Legvold, in Ferromagnetic Materials, edited by E. P. Wohlfarth (North-Holland, Amsterdam, 1980), Vol. 1, pp. 183-295.

${ }^{7}$ J. M. D. Coey, V. Skumryev, and K. Gallagher, Nature (London) 401, 35 (1999).

${ }^{8}$ S. N. Kaul and S. Srinath, Phys. Rev. B 62, 1114 (2000).

${ }^{9}$ I. D. Hughes, M. Däne, A. Ernst, W. Hergert, M. Lüders, J. Poulter, J. B. Staunton, A. Svane, Z. Szotek, and W. M. Temmerman, Nature (London) 446, 650 (2007).

${ }^{10}$ S. Srinath and S. N. Kaul, Phys. Rev. B 60, 12166 (1999).

${ }^{11}$ C. Santos, W. Nolting, and V. Eyert, Phys. Rev. B 69, 214412 (2004).

${ }^{12}$ K. M. Döbrich, A. Bostwick, E. Rotenberg, and G. Kaindl, Phys. Rev. B 81, 012401 (2010).

${ }^{13}$ S. Y. Dan'kov, A. M. Tishin, V. K. Pecharsky, and K. A. Gschneidner Jr., Phys. Rev. B 57, 3478 (1998).

${ }^{14}$ J. Lyubina, M. D. Kuz'min, K. Nenkov, O. Gutfleisch, M. Richter, D. L. Schlagel, T. A. Lograsso, and K. A. Gschneidner Jr., Phys. Rev. B 83, 012403 (2011).

${ }^{15}$ M. Colarieti-Tosti, S. I. Simak, R. Ahuja, L. Nordström, O. Eriksson, D. Åberg, S. Edvardsson, and M. S. S. Brooks, Phys. Rev. Lett. 91, 157201 (2003).

${ }^{16} \mathrm{M}$. Farle, K. Baberschke, U. Stetter, A. Aspelmeier, and F. Gerhardter, Phys. Rev. B 47, 11571 (1993).

${ }^{17}$ M. Gajdzik, T. Trappmann, C. Sürgers, and H. v. Löhneysen, Phys. Rev. B 57, 3525 (1998).

${ }^{18}$ D. Michels, C. E. Krill III, and R. Birringer, J. Magn. Magn. Mater. 250, 2003 (2002).

${ }^{19}$ R. Kruk, M. Ghafari, H. Hahn, D. Michels, R. Birringer, C. E. Krill III, R. Kmiec, and M. Marszalek, Phys. Rev. B 73, 054420 (2006).

${ }^{20}$ M. Yue, J. X. Zhang, H. Zeng, and K. J. Wang, Appl. Phys. Lett. 89, 232504 (2006).

${ }^{21}$ P. M. Shand, J. G. Bohnet, J. Goertzen, J. E. Shield, D. Schmitter, G. Shelburne, and D. L. Leslie-Pelecky, Phys. Rev. B 77, 184415 (2008).

${ }^{22}$ S. N. Kaul and S. P. Mathew, Phys. Rev. Lett. 106, 247204 (2011).

${ }^{23}$ S. P. Mathew and S. N. Kaul, Appl. Phys. Lett. 98, 172505 (2011).

${ }^{24}$ G. Will, R. Nathans, and H. A. Alperin, J. Appl. Phys. 35, 1045 (1964).

${ }^{25}$ J. W. Cable and E. O. Wollan, Phys. Rev. 165, 733 (1968).

${ }^{26}$ W. C. Koehler, H. R. Child, R. M. Nicklow, H. G. Smith, R. M. Moon, and J. W. Cable, Phys. Rev. Lett. 24, 16 (1970).

${ }^{27}$ R. M. Moon, W. C. Koehler, J. W. Cable, and H. R. Child, Phys. Rev. B 5, 997 (1972).

${ }^{28}$ H. R. Child, Phys. Rev. B 18, 1247 (1978).

${ }^{29}$ A. Michels, F. Döbrich, M. Elmas, A. Ferdinand, J. Markmann, M. Sharp, H. Eckerlebe, J. Kohlbrecher, and R. Birringer, Europhys. Lett. 81, 66003 (2008).

${ }^{30}$ F. Döbrich, M. Elmas, A. Ferdinand, J. Markmann, M. Sharp, H. Eckerlebe, J. Kohlbrecher, R. Birringer, and A. Michels, J. Phys. Condens. Matter 21, 156003 (2009).

${ }^{31}$ R. Birringer, H. Gleiter, H.-P. Klein, and P. Marquardt, Phys. Lett. A 102, 365 (1984).
${ }^{32}$ J. Markmann, V. Yamakov, and J. Weissmüller, Script. Mater. 59, 15 (2008).

${ }^{33}$ H. P. Klug and L. E. Alexander, X-Ray Diffraction Procedures for Polycrystalline and Amorphous Materials (Wiley, New York, 1974), Chap. 9.

${ }^{34}$ V. F. Sears, Neutron News 3, 26 (1992).

${ }^{35}$ Note that the actual thicknesses $h$ of the two utilized ${ }^{160} \mathrm{Gd}$ samples, $275 \mu \mathrm{m}$ and $318 \mu \mathrm{m}$, result in a scattering intensity $I_{S}$ that is close to the optimum value since, neglecting attenuation due to scattering, $I_{S} \propto h \exp \left(-\rho \sigma_{a} h\right)$ holds. ${ }^{39}$ Here, $\rho$ denotes the atomic density.

${ }^{36}$ H. Berger, Annu. Rev. Nucl. Sci. 21, 335 (1971).

${ }^{37}$ C. J. Glinka, J. G. Barker, B. Hammouda, S. Krueger, J. J. Moyer, and W. J. Orts, J. Appl. Crystallogr. 31, 430 (1998).

${ }^{38}$ G. D. Wignall and F. S. Bates, J. Appl. Crystallogr. 20, 28 (1987).

${ }^{39}$ Small-Angle X-Ray Scattering, edited by O. Glatter and O. Kratky (Academic, London, 1982).

${ }^{40}$ A. Wiedenmann, in Neutron Scattering from Magnetic Materials, edited by T. Chatterji (Elsevier, Amsterdam, 2006), pp. 473-520.

${ }^{41}$ A. Michels and J. Weissmüller, Rep. Prog. Phys. 71, 066501 (2008).

${ }^{42}$ J. Weissmüller, A. Michels, D. Michels, A. Wiedenmann, C. E. Krill III, H. M. Sauer, and R. Birringer, Phys. Rev. B 69, 054402 (2004).

${ }^{43}$ A. Michels, C. Vecchini, O. Moze, K. Suzuki, P. K. Pranzas, J. Kohlbrecher, and J. Weissmüller, Phys. Rev. B 74, 134407 (2006).

${ }^{44}$ A. Michels, R. N. Viswanath, J. G. Barker, R. Birringer, and J. Weissmüller, Phys. Rev. Lett. 91, 267204 (2003).

${ }^{45}$ A. Michels, Phys. Rev. B 82, 024433 (2010).

${ }^{46}$ B. E. Warren, X-Ray Diffraction (Dover, New York, 1990).

${ }^{47}$ D. Michels, Ph.D. thesis, Universität des Saarlandes, 2005.

${ }^{48}$ J. Weissmüller, A. Michels, J. G. Barker, A. Wiedenmann, U. Erb, and R. D. Shull, Phys. Rev. B 63, 214414 (2001).

${ }^{49}$ E. E. Underwood, Quantitative Stereology (Addison-Wesley, Reading, MA, 1970).

${ }^{50}$ G. Herzer, in Handbook of Magnetic Materials, edited by K. H. J. Buschow (Elsevier, Amsterdam, 1997), Vol. 10, pp. 415-462.

${ }^{51}$ S. P. Mathew, S. N. Kaul, A. Nigam, A.-C. Probst, and R. Birringer, J. Phys. Conf. Ser.200, 072047 (2009).

${ }^{52}$ R. Skomski, H. Zeng, and D. J. Sellmyer, IEEE Trans. Magn. 37, 2549 (2001).

${ }^{53}$ J. F. Löffler, H. B. Braun, W. Wagner, G. Kostorz, and A. Wiedenmann, Phys. Rev. B 71, 134410 (2005).

${ }^{54}$ A. Michels, M. Elmas, F. Döbrich, M. Ames, J. Markmann, M. Sharp, H. Eckerlebe, J. Kohlbrecher, and R. Birringer, Europhys. Lett. 85, 47003 (2009).

${ }^{55}$ J. F. Löffler, H. B. Braun, and W. Wagner, Phys. Rev. Lett. 85, 1990 (2000).

${ }^{56}$ G. E. Bacon, Neutron Diffraction (Clarendon, Oxford, 1962).

${ }^{57}$ E. P. Wohlfarth, in Ferromagnetic Materials, edited by E. P. Wohlfarth (North-Holland, Amsterdam, 1980), Vol. 1, pp. $1-70$.

${ }^{58} \mathrm{~A}$. Aharoni, Introduction to the Theory of Ferromagnetism, 2nd ed. (Clarendon, Oxford, 1996).

${ }^{59}$ M. Bachmann, R. Fischer, and H. Kronmüller, in Magnetic Anisotropy and Coercivity in Rare-Earth Transition Metal Alloys, edited by L. Schultz and K.-H. Müller (WerkstoffInformationsgesellschaft, Frankfurt, 1998), pp. 217-236. 
${ }^{60} \mathrm{H}$. Kronmüller and M. Fähnle, Micromagnetism and the Microstructure of Ferromagnetic Solids (Cambridge University Press, Cambridge, 2003).

${ }^{61}$ The lattice parameters of hcp Gd at $T=300 \mathrm{~K}$ are $a=3.63 \AA$ and $c=5.78 \AA$ (Ref. 72).

${ }^{62}$ T. Schrefl, J. Fidler, and H. Kronmüller, Phys. Rev. B 49, 6100 (1994).

${ }^{63} \mathrm{~K}$. A. McEwen, in Handbook on the Physics and Chemistry of Rare Earths, edited by K. A. Gschneidner and L. Eyring (North-Holland, Amsterdam, 1978), Vol. 1, pp. 413-485.

${ }^{64}$ M. S. S. Brooks and D. A. Goodings, J. Phys. C 1, 1279 (1968).

${ }^{65}$ Note that no assumption is made regarding the nature or origin of the magnetization reduction at interfaces. In fact, one may as well consider an enhanced grain-boundary magnetization, although we are not aware of any material exhibiting such a property.

${ }^{66}$ Note that, for case of Gd, we do not expect a change of the atomic magnetic moment in the vicinity of lattice defects since the magnetism of Gd arises predominantly from the spin moments of the localized $4 f$ electrons. ${ }^{6}$ However, as discussed in Sec. IV B, atomic- site disorder at grain boundaries may lead to a modified interatomic exchange coupling and thus a reduced effective magnetization in the core region of the grain boundaries.

${ }^{67}$ C. E. Krill and R. Birringer, Philos. Mag. A 77, 621 (1998).

${ }^{68}$ M. Ames, J. Markmann, R. Karos, A. Michels, A. Tschöpe, and R. Birringer, Acta Mater. 56, 4255 (2008).

${ }^{69}$ Since the core region of grain boundaries embodies excess volume, resulting in a local density deficit of typically 5\% (see Ref. 73), the contribution of grain-boundary excess volume to a reduced overall density is, for a given crystallite size of $20 \mathrm{~nm}$, approximately $1 \%$.

${ }^{70} \mathrm{~J}$. P. Hirth and J. Lothe, Theory of Dislocations (Wiley, New York, 1982).

${ }^{71}$ In a lattice with cubic symmetry, one analyzes reflections corresponding to the same crystallographic direction. This is also possible in hcp materials looking in the $c$ direction, i.e., at the (002) and the (004) peaks.

${ }^{72}$ F. J. Darnell, Phys. Rev. 130, 1825 (1963).

${ }^{73}$ C. E. Krill III, L. Helfen, D. Michels, H. Natter, A. Fitch, O. Masson, and R. Birringer, Phys. Rev. Lett. 86, 842 (2001). 